Check for updates

Cite this: RSC Adv., 2017, 7, 36949

Received 8th March 2017 Accepted 29th May 2017 DOI: $10.1039 / c 7 r a 02845 h$

rsc.li/rsc-advances

\section{Recent advancements, key challenges and solutions in non-enzymatic electrochemical glucose sensors based on graphene platforms}

\author{
G. Gnana kumar, (DD *a G. Amala and S. M. Gowtham ${ }^{\text {b }}$
}

The increasing demand for the development of highly sensitive, selective and precise non-enzymatic electrochemical sensors (NEGS) for the quantitative and qualitative analysis of glucose in clinical, pharmaceutical and industrial sectors has garnered attention worldwide. The electroactive materials are catalytically oxidized glucose molecules that generate signals for glucose quantification; graphene and its derivatives are highly useful as active conductive supports for high-performance NEGS. Since the discovery of graphene in 2004, the astonishing properties of graphene-based platforms have resulted in its extensive applications in NEGS. Although a number of research efforts have involved the development of NEGS with graphene and its composites, a comprehensive study of NEGS using graphene and its composites has yet to be envisioned; this has not only obscured their widespread application but has also diminished future improvements in NEGS. Hence, this review article is presented to visualize the significant requirements of NEGS catalysts, the influences of graphene in achieving the requirements of NEGS catalysts and the progress made on graphene platform-equipped NEGS, along with a detailed discussion of recent studies and the involved electrochemical mechanisms. Building on the above perspectives, the fine tuning of the sensitivities and detection limits of graphene-based platforms in the fabrication of NEGS have also been detailed. Furthermore, this article clearly outlines future directions by detailing the obstacles currently facing grapheneequipped NEGS; as such, this review article can be considered to be a useful guide in the design and development of next-generation NEGS.
aDepartment of Physical Chemistry, School of Chemistry, Madurai Kamaraj University, Madurai 625 021, Tamil Nadu, India. E-mail: kumarg2006@gmail.com
${ }^{b}$ School of Chemistry, Madurai Kamaraj University, Madurai 625 021, Tamil Nadu, India

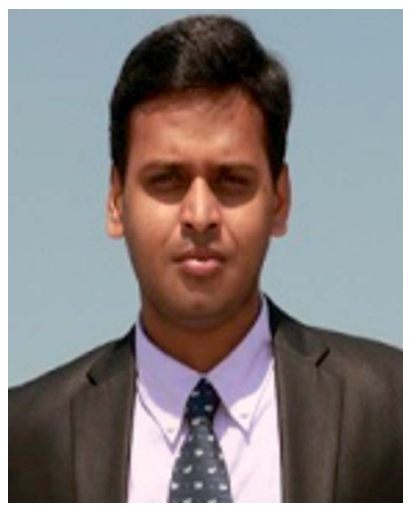

Dr G. Gnana kumar is working as an Assistant Professor in the Department of Physical Chemistry, School of Chemistry, Madurai Kamaraj University, Madurai, India. He has published more than 90 articles in reputed scientific journals and has contributed chapters to books. His main research areas include the design and development of grapheme-based electrode materials, graphenesupported electrocatalysts and polymer nanocomposites applicable for a number of electrochemical applications, including electrochemical sensors, fuel cells, solar cells, and lithium-air batteries.

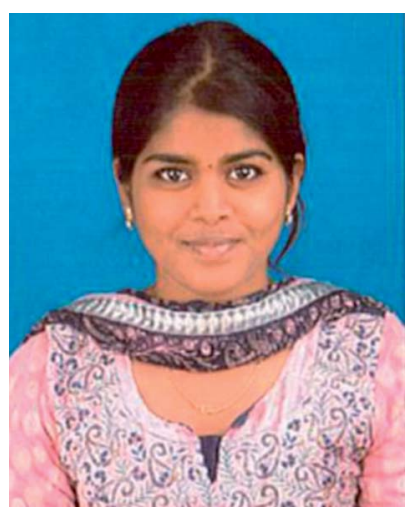

Ms. G. Amala obtained her M.Sc. degree at Madurai Kamaraj University, Madurai, India under the guidance of $\mathrm{Dr}$ G. Gnana Kumar, focusing on graphenebased nanocatalysts for sensitive and selective non-enzymatic glucose sensors. Since completing her M.Sc., she is focusing on the development of electroorganic materials for nonenzymatic glucose sensor applications for her doctoral studies. 


\section{Introduction}

\subsection{Significance of glucose monitoring}

Glucose is a highly important corporal analyte that is involved in major catabolic pathways, including glycolysis and oxidative phosphorylation. It has been described as a potential stockpile for cells and is the only energy supply for brain and red blood cells. ${ }^{1}$ It is currently understood that glucose is synthesized in the digestive system and absorbed through the intestines. Habitually, the body consumes free glucose and converts it into the more storable form of glycogen, which is then stored in the liver, muscles and tissues. ${ }^{2,3}$ These processes are restrained by a set of metabolic hormones that can be viewed as dual aspects concerning glucose regulation; the first is insulin, which is created by the $\beta$-cells in the pancreas and is primarily responsible for revamping freshly created glucose into a storable format, glycogen, for further use. The other is glucagon, which is released by pancreatic $\alpha$-cells and triggers the liver and muscles to release glycogen and convert it into usable glucose. ${ }^{4}$

The normal blood glucose level (tested while fasting) of humans ranges between 3.9 and $5.5 \mathrm{mmol} \mathrm{L}^{-1}$ (70 to $100 \mathrm{mg}$ $\left.\mathrm{dL}^{-1}\right){ }^{5}$ blood sugar levels outside this range indicate abnormalities. A high level of glucose is referred to as hyperglycemia, while diabetes mellitus (DM) is characterized by persistent hyperglycemia and is the most deadly disease related to poor blood sugar regulation. The blood glucose level for this abnormality ranges between 5.6 and $7 \mathrm{mmol} \mathrm{L}^{-1}$ (100 to $126 \mathrm{mg}$ $\left.\mathrm{dL}^{-1}\right){ }^{6}$ In general, DM is divided into three types: Type 1 , Type 2 and gestational diabetes. Type 1-DM, where the pancreases either loses its ability to produce insulin or produces minimal amounts, is increasing among young people; $10 \%$ of diabetic patients have this type of diabetes. Middle-aged or older people are affected by Type 2-DM, which is either due to the nonproduction of influential insulin or the inability to effectively utilize insulin; $90 \%$ of diabetic patients are in this category. ${ }^{7,8}$ Diabetes generated during pregnancy is termed gestational diabetes; this affects $2 \%$ to $4 \%$ of pregnancies, affecting both mother and child. ${ }^{9}$ The prevalence of diabetes represents a major health problem that ranks $8^{\text {th }}$ among the most deadly diseases; globally, around 9\% of adults suffer from anomalous blood glucose levels (as per WHO, 2014). ${ }^{\mathbf{1 0 1 1}}$ On the other hand,

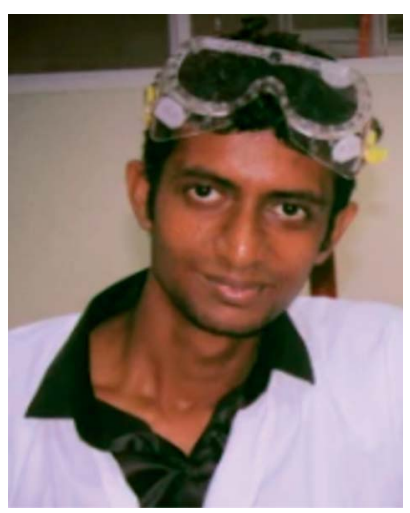

Mr. S. M. Gowtham obtained his M.Sc. degree in Chemistry from Madurai Kamaraj University, Madurai, Tamil Nadu, India, in 2013. His current research interest lies in the graphene based nanomaterials and core-shell nanostructures for biosensor applications. a dramatic drop in blood sugar level $\left(3.9 \mathrm{mmol} \mathrm{L}^{-1}(70 \mathrm{mg}\right.$ $\left.\mathrm{dL}^{-1}\right)$ ) leads to a potentially fatal condition called hypoglycemia. $^{12}$ Owing to glucose level fluctuations, symptoms including lethargy, impaired mental functioning, irritability, sweating, paranoid or aggressive mentality, and loss of consciousness, which eventually decrease the lifespan of humans. ${ }^{13}$ The challenge of controlling diabetes is strongly associated with the reliable, accurate, rapid, sensitive and attentive monitoring of glucose levels, and related research activities remain a subject of exploration.

\subsection{Importance of NEGS in glucose level quantification}

For the above reasons, a number of techniques, including colorimetry, ${ }^{\mathbf{1 4}}$ optical transduction, ${ }^{15}$ microdialysis, ${ }^{\mathbf{1 6}}$ electrochemiluminescence, ${ }^{17}$ reverse iontophoresis, ${ }^{18}$ and sonophoresis, ${ }^{19}$ have been exploited for glucose quantification. However, the aforesaid techniques display certain constraints, including matrix interference, high cost, tedious fabrication processes, lower reproducibility, and shorter shelf-time; this necessitates the exploration of efficient techniques for glucose quantification. Recently, electrochemical techniques have received keen attention in glucose sensors owing to their accuracy, specificity, rapidity, simplicity, better detection limits, real time detection, elevated electron transfer kinetics, high chemical stability, comparable, biocompatibility, possibility of scale-up processes, etc. $^{\mathbf{2 0 , 2 1}}$ Electrochemical glucose sensors are generally sorted into two categories, viz. enzymatic (EGS) and non-enzymatic sensors (NEGS). Glucose quantification by coupling with an enzyme as a molecular-recognition element has been greatly studied for applications in enzymatic glucose sensors, and glucose oxidase and glucose dehydrogenase-based sensors have seized a large portion of the market. ${ }^{22}$ However, EGS exhibits certain limitations from the point of view of enzymes and remains a developing technology due to the denaturation of enzymes, inefficient electron transfer between the electrode surface and the enzymes, tedious immobilization techniques (adsorption, entrapment, covalent linking and cross linking), thermal and chemical deformation, poor reproducibility, insufficient stability and $\mathrm{pH}$, and humidity and temperature dependence, all of which affect the EGS performance. ${ }^{23}$ The aforesaid drawbacks of EGSs can be effectively addressed by catalyzed electrochemical processes in the form of NEGS, which is an interesting alternative to conventional techniques.

\subsection{History of NEGS and requirement of graphene-based platforms for NEGS}

The history of NEGS originates in 1909, when the direct electrooxidation of glucose into gluconic acid was achieved at a lead anode under acidic conditions. ${ }^{24}$ The sluggish electrokinetics exhibited by conventional electrodes toward glucose oxidation was improved by nanometric catalysts, and the oxidation of analytes has been achieved at the active sites of catalysts under neutral/alkaline/acidic conditions. Later, the electrochemical detection of glucose was catalytically improved with a number of zero dimensional (OD) and one dimensional (1D) nanomaterials, including nanoparticles, ${ }^{25}$ quantum dots, ${ }^{26}$ 
nanowires ${ }^{27}$ and carbon nanotubes ${ }^{28}$ The greatest challenge of NEGS is durability, which can be effectively overcome by the aforementioned nanometric catalysts; however, the intermediate products of glucose oxidation adsorbed over the electrode surface eventually limit its sensitivity.$^{29}$ In addition, the inferior selectivity and high chloride poisoning exhibited by NEGS in comparison with EGS remain great obstacles to the commercialization of NEGS; $;^{30}$ this necessitates the immense exploration and development of active carbon materials which can be uniformly implant the active sites and can preserve during repetitive cycles under harsh electrochemical regimes and conditions. Recently, two dimensional (2D) and three dimensional (3D) graphene, consisting of single-layer sheets with $\mathrm{sp}^{2}$ hybridized carbon atoms, which is adjacently packed into a hexagonal lattice, has attracted attention for utilization in NEGS owing to its beneficial physio and electrochemical properties. The exceptional and tunable electrical properties of graphene, including its band gap, charge carrier type, concentration, mobility and density (field effect) and electron transfer rate, have enabled its applications in electrochemical devices. The influential properties of graphene, such as its elevated theoretical surface area of $\approx 2630 \mathrm{~m}^{2} \mathrm{~g}^{-1}$, large potential window, depressed charge-transfer resistance, high robustness and flexibility, enable its application as an ideal electrode material. ${ }^{31}$ Furthermore, graphene-based platforms serve as effective nucleation centres for the homogeneous distribution of active sites and preserve their electrocatalytic activities over repetitive cycles under harsh electrochemical regimes and conditions; this addresses the inferior sensitivity, selectivity and chloride poisoning constraints of conventional NEGS and affirms the significance of graphene-based platforms in the fabrication of NEGS. Despite the vigorous research efforts devoted to graphene, efficient graphene catalyst-based portable NEGS have not yet been developed; this has not only decreased the widespread application of graphene but also the large scale applications of NEGS. While a few review articles have described the utilization of graphene platforms in chemical sensors and biosensors, these reviews exclusively focus on the preparation of new graphene-based catalysts applicable for NEGS; however, our understanding is limited on the function of these materials, the pertinent mechanisms involved in catalyst preparation, the strategies behind the utilization of active carbon-based catalysts, the underlying detection mechanisms, the recent advancements, and the key challenges and solutions of graphene platform-equipped NEGS. If comprehensive efforts and advanced ideas could be brought forward to improve our understanding of the aforementioned significant issues, the real-time application of NEGS could be realized. Hence, this review is presented to visualize the significant requirements of NEGS catalysts, the influence of graphene in achieving the requirements of catalytic probes, and the progress made on graphene platform-equipped NEGS; it includes a detailed discussion of recent research efforts along with the involved electrochemical mechanisms, which cannot be clearly understood from previous reviews. Building on the above perspectives, the fine-tuning of the sensitivity and detection limits of graphene-based platforms in the fabrication of NEGS has also been detailed. Furthermore, this article clearly outlines future research directions by detailing the present obstacles facing graphene-equipped NEGS; as such, this review article can be considered as a useful guide for the design and development of next-generation NEGS. Hence, the main objective of this review is to provide an overview of the challenges of existing NEGS, the requirements of graphene utilization, the research efforts devoted to graphene-based catalysts and compatible solutions for the development of electrochemical devices with advanced features.

\section{Mechanisms involved in NEGS}

In NEGS, electrode surfaces modified with active nanomaterials serve as catalysts for the electrooxidation of glucose; two mechanisms have been widely studied to explain the aforementioned process. The activated chemisorption model proposed by Pletcher assumes that the analyte glucose is adsorbed on the electrode surface via the unpaired d-electrons and unfilled d-orbitals of transition metal atoms, which generates bonds with the adsorbates. ${ }^{32}$ This is followed by simultaneous hydrogen abstraction and adsorption of the organic species, leading to the electrocatalysis of glucose oxidation; the abstraction of hydrogen is considered to be the rate-determining step (Fig. 1a). However, the adsorption model has failed to explain the role of hydroxyl radicals generated in alkaline medium. It has already been proven that glucose electrooxidation can occur via adsorbed hydroxyl groups $\left(\mathrm{OH}_{\mathrm{ads}}\right)$. The "Incipient Hydrous Oxide Adatom Mediator" (IHOAM) model introduced by Bruke specifies that a formation of a premonolayer at the active metal atoms determines the glucose electrooxidation process at the NEGS. ${ }^{33}$ The generated $\mathrm{OH}_{\text {ads }}$ layer serves as both a mediator and inhibitor for the oxidation and reduction processes. The chemisorption of

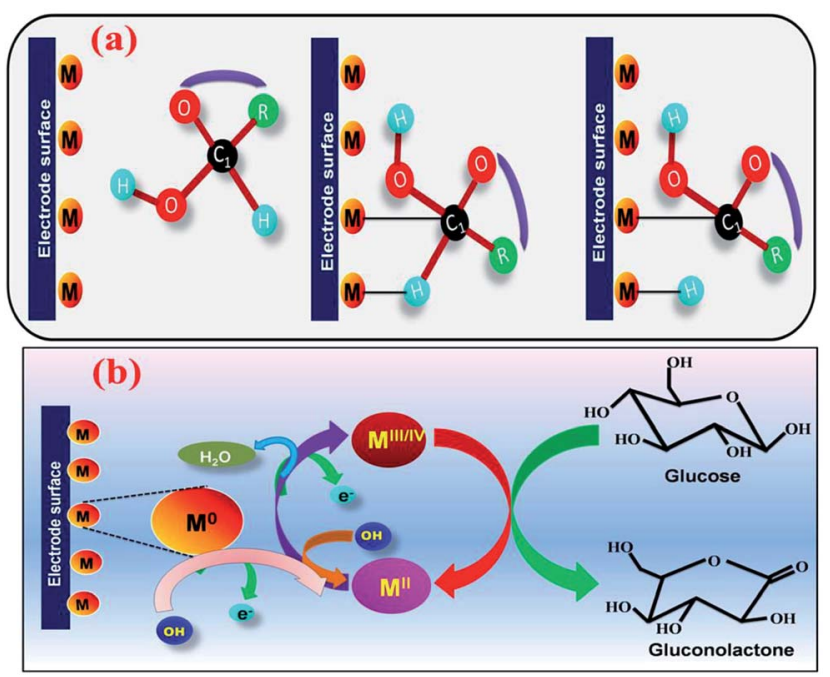

Fig. 1 (a) The activated chemisorption model associated in the glucose electrooxidation at metal active centres (redrawn with permission from Springer; Pletcher, 1984) and (b) the IHOAM model of the electrooxidation of glucose at metal active centres under alkaline conditions (redrawn with permission from Elsevier; Burke, 1994). 
hydroxide anions on the reductive metal adsorption sites leads to the formation of $\mathrm{MOH}_{\mathrm{ads}}$, which is then electrocatalytically oxidized the glucose. Hence, it is clear that the formation of the $\mathrm{MOH}_{\text {ads }}$ species is purely dependent upon the alkaline environment, leading to the occurrence of more electrooxidation reactions in higher $\mathrm{pH}$ environments (Fig. 1b).

\section{The influence of graphene in achieving the requirements of catalysts applicable for NEGS}

\subsection{Adsorption of an analyte}

In general, the activity of a NEGS is initiated at the catalystmodified electrode via the adsorption and diffusion of glucose followed by electrochemical oxidation. The efficient adsorption and diffusion facilitates the direct contact of the analyte with the electrochemically active sites, where the initial electron transport is caused by the uniform adsorption of an analyte. The adsorption rate of an analyte is purely dependent upon the active surface area (ASA) of an electrocatalyst and can be effectively increased by its porosity, surface smoothness and unique morphological features. ${ }^{34}$ Although the electrochemical oxidation of glucose is influenced by nanometric metal catalysts, their agglomeration in an aqueous environment reduces the ASA, which may limit the electrochemical performance of NEGS. ${ }^{35}$ The adsorption of an analyte can be increased by optimizing the pore properties of catalysts; the pores should not only be tuned to adsorb the analyte but also to retain the analyte in its active channels for durable NEGS performance. Hence, the exploitation of active support materials, in which graphene is vital, to prevent the loss of the ASA of nanoparticles has received considerable attention. The active graphene comprises a theoretical specific surface area of $2630 \mathrm{~m}^{2} \mathrm{~g}^{-1}$, which is superior to that of carbon nanotubes (CNTs) $\left(50\right.$ to $\left.1315 \mathrm{~m}^{2} \mathrm{~g}^{-1}\right)$, owing to its $2 \mathrm{D}$ honeycomb lattice nanosheet structure with $\mathrm{sp}^{2}$ bonded carbon atoms. ${ }^{36}$ Furthermore, the adsorption of an analyte and its effectual retention can be increased by stacking layers of graphene. Although the accumulation of nanoparticles on the graphene sheets reduces the porosity of the composite in comparison with the individual components, the effectual adsorption can be balanced with the increased surface area provided via the synergetic effects of graphene and metal nanoparticles. $^{37}$

\subsection{Electrical conductivity}

The mechanism of electrochemical glucose oxidation is purely governed by the interfacial contact between the current collector and the electroactive material. ${ }^{38}$ The electrooxidation of glucose experiences sluggish electrokinetics at conventional bare electrodes owing to the large overpotential achieved via its limited electrical conductivity; this necessitates modification of the electrode materials with highly conductive catalysts. The electrical conductivity of the catalyst serves as an electronic wire to endorse electron transfer between the catalytic active centres and the surface of a current collector. The interfacial contact can be ultimately improved by decreasing the interfacial charge resistance (ICR) and large overpotential via electrically conductive materials. ${ }^{39}$

Effective interfacial contact can be achieved using active carbon materials including graphene, graphene oxide (GO) and reduced graphene oxide (rGO) owing to their high electrical conductivities and versatile structural features. Graphene has a unique 2D structure with one-atom thickness which provides remarkably enhanced charge mobility in the range of $10^{6} \mathrm{~cm}^{2}$ $\mathrm{V}^{-1} \mathrm{~s}^{-1}$ (higher than $\mathrm{Si}$ ) and high electrical conductivity of 7200 $\mathrm{S} \mathrm{cm}{ }^{-1}$, which is approximately 60 times higher than that of single walled CNTs (SWCNTs) (3000 to $\left.4000 \mathrm{~S} \mathrm{~cm}^{-1}\right) .{ }^{40}$ The carrier mobility of exfoliated graphene is found to be 100000 $\mathrm{cm}^{2} \mathrm{~V}^{-1} \mathrm{~s}^{-1}$, which is also higher than that of SWCNTs $\left(\approx 80000 \mathrm{~cm}^{2} \mathrm{~V}^{-1} \mathrm{~s}^{-1}\right)$; this demonstrates the high speed electronics of graphene platforms. ${ }^{40}$ At room temperature, graphene displays an elevated thermal conductivity $\left(5300 \mathrm{~W} \mathrm{~m}^{-1}\right.$ $\left.\mathrm{K}^{-1}\right)$, which is higher than that of SWCNTs $\left(3500 \mathrm{~W} \mathrm{~m}^{-1} \mathrm{~K}^{-1}\right) .{ }^{40}$ Graphene reacts like a metal, with almost constant and high mobility over a large range of temperatures and charge densities owing to the $2 \mathrm{D} / 3 \mathrm{D}$ conductive network of charge carriers over its surface. ${ }^{41}$ These elite electrical properties of graphene and its derivatives are attributed to their Dirac points and honeycomb lattice with a highly interconnected carbon atom matrix, where the energy bands of one carbon atom are interconnected with another in turn to provide a rapid flow of electrons in the range of the speed of light. The $\pi-\pi$ conjugation of $\mathrm{sp}^{2}$ hybridized carbon atoms of graphene delivers higher electron mobility without barriers over the whole layer structure of the graphene sheets. ${ }^{42}$ Furthermore, electrochemical sensors with graphene have low residual currents, prompt sustainable surfaces, excellent carrier mobility and capacitance, elevated electron transfer rates, robustness and flexibility; thus, they are highly promising candidates to fulfil the requirements of highperformance NEGS.

\subsection{Stability}

Real-time benefits of NEGS can be realized due to their durability, which is governed by the robust hydrolytic, thermal, mechanical, electrooxidative and reductive stabilities of their catalysts under harsh electrochemical regimes and conditions. ${ }^{43}$ Hydrolytic stability results in a stable electroactive material in an aqueous environment, and agglomeration may effectively decrease glucose sensing performance via reduced electrochemical ASA (EASA). ${ }^{44}$ This drawback can be effectively overcome by graphene platforms, and the agglomeration of bare metal nanoparticles and restacking of graphene can be addressed by effectively piling the metal nanoparticles over the large surface of graphene, which retains the large surface area of the composite even under harsh electrochemical regimes and conditions. Furthermore, graphene exhibits an optimal mechanical stability (130 GPa), higher thermal stability and greater level of flexibility owing to its highly interconnected honeycomb latticed $\mathrm{sp}^{2}$ atomic structure with carbon bonds of $0.142 \mathrm{~nm}$ in length, which would be useful to develop robust NEGS probes for applications in on-site detection. ${ }^{45}$ 


\subsection{Biocompatibility}

Successful glucose monitoring can be accomplished using biocompatible catalysts, owing to the direct contact of the fabricated sensors with real samples, including blood, serum, urine, etc. ${ }^{46}$ Real-time glucose detection can be achieved via two methods: external analysis of glucose levels with samples collected from patients, and pricking patients with the fabricated NEGS. In both methods, the cellular organs of the biological samples cannot be affected by the cytotoxic effects of the used catalysts; the influence of the toxicity of the catalysts will be greater in the second case, which involves direct exposure of the patients to the NEGS. Hence, it is clear that the active catalyst should not only be designed from the perspective of electrochemical properties, but also with the aim of preventing the triggering of biological reactions and damaging the cells in bio-samples.

Although carbon-based materials may not lead to any biological reactions owing to their inert behavior, their toxicity must be considered for on-site applications. In general, CNTs are prepared using metallic nanoparticles as templates from carbon-containing gases, which results in the existence of metallic impurities. The aforementioned metallic impurities are electrochemically active and strongly affect the electrochemistry of the CNTs. Direct exposure to CNTs causes adverse effects, including lung inflammation, cardiovascular diseases, and skin cell damage, owing to the participation of metallic impurities in redox reactions with active biomolecules. In comparison with CNTs, graphene exhibits lower toxicity owing to its unique structural features, such as a smooth surface, oxygen functionalities with smooth edges, and the absence of metallic impurities. ${ }^{47}$ The biocompatibility of graphene has already been actualized using biological molecules, such as deoxyribonucleic acid (DNA), proteins, and peptides, on its surface. ${ }^{\mathbf{4 8 - 5 0}}$ Furthermore, graphene provides greater affinity for the attachment and growth of cellular biomaterial and provides a sufficiently biocompatible environment owing to the lack of biological toxicity. The biocompatibility of graphene can be further improved with nitrogen doping because the $\mathrm{C}-\mathrm{N}$ microenvironment has lower cytotoxicity and high biocompatibility, where biological bases are nitrogenous complexes. ${ }^{51}$ Furthermore, the conversion of $2 \mathrm{D}$ graphene to a $3 \mathrm{D}$ structure may lead to enhanced biological compatibility due to its high surface network, porous compartments and enormous charge transformation ability. The utilization of biocompatible agents such as bio-mass extracts, microorganisms, and ionic liquids for the reduction of GO will effectively replace toxic reducing agents including hydroquinone, hydrazine hydrate, and dimethyl hydrazine and may enable the introduction of environmentally benign surfaces onto graphene platforms.

\subsection{Antipoisoning and antifouling effects}

In real sample analysis, the cellular organs, proteins and other interfering species present in bio-samples may accumulate on the surfaces of electrodes, leading to surface fouling effects; this suppresses the sensitivity and long-term stability of the electrodes via disrupting the interpermeation of the desired molecules. ${ }^{52}$ Active graphene serves as an effective antifouling material with negative charge, hydrophilicity and surface smoothness; it may repel the negatively charged and hydrophilic foulants and effectively prevent the loss of sensitivity. ${ }^{53}$ The adsorbed intermediates and poisoning by chloride ions over the active sites of the electrocatalyst result in poisoning of the electrocatalyst, where the active sites of the catalyst adsorb intermediate species and chloride ions. When the poisoning intermediates are further electrochemically oxidized, the EASA and the active sites of the catalysts dramatically decrease, which decreases the sensitivity of the fabricated NEGS. This drawback can be effectively overcome by graphene owing to its large surface area and edge-plane defect sites. The electrooxidative and reductive stabilities of catalysts can be ensured by the porous structure of graphene, offering easier pathways for the escape of oxygen gas bubbles during the electrochemical process. When the electrochemical probe faces real biological samples, it must confront numerous electroactive interfering species, such as uric acid (UA), ascorbic acid (AA), dopamine (DA) and other carbohydrates, along with the major components of glucose ( 3 to $8 \mathrm{mM}){ }^{54}$ The aforementioned interfering species may effectively influence the sensitivity of a fabricated NEGS; this can be overcome with the aid of effective charge transfer via graphene, which may retain sensitivity for multple analyses.

\subsection{Cost}

Although immense research interest was inspired by NEGS a century ago, more than $\mathbf{4 1 5}$ million diabetic patients are still seeking affordable and efficient glucose sensors. ${ }^{55}$ Currently, the cost of commercialized bio-probe-assisted glucometers based on EGS varies from $\$ 80$ to $\$ 1400$ on account of their expensive enzymatic systems $(1 \mathrm{~g}$ glucose oxidase $=\$ 475)$ and tedious enzyme immobilization techniques, which limits their extensive utilization. ${ }^{56}$ Although NEGS effectively reduce the cost of currently available sensors, their cost is entirely dominated by their catalysts. In general, CNTs are prepared via chemical-vapour deposition (CVD) using carbon-containing gases, high temperatures and sophisticated instruments, which has increased the production cost of CNTs. ${ }^{40}$ In contrast, the production costs of graphene-based platforms are affordable due to their inexpensive and accessible main source material, graphite. ${ }^{40}$ The conjugation of graphene material in NEGS can effectively overcome the aforementioned limitations with the utilization of lower amounts of catalysts, which may provide affordable, relevant and reliable NEGS. Although the utilization of graphene-based NEGS is restricted by the high cost of graphene synthesis achieved via sophisticated techniques, including chemical vapour deposition, epitaxial growth, and exfoliation techniques, this restriction can be effectively overcome by using graphene derivatives such as GO and rGO. Specifically, rGO has been extensively utilized in NEGS owing to its unique properties and its similar configuration to that of graphene. Green synthesis routes for the reduction of GO associated with scalable biomass, microorganisms, sunlight, etc., may further lower the overall cost of rGO synthesis. ${ }^{57,58}$ 


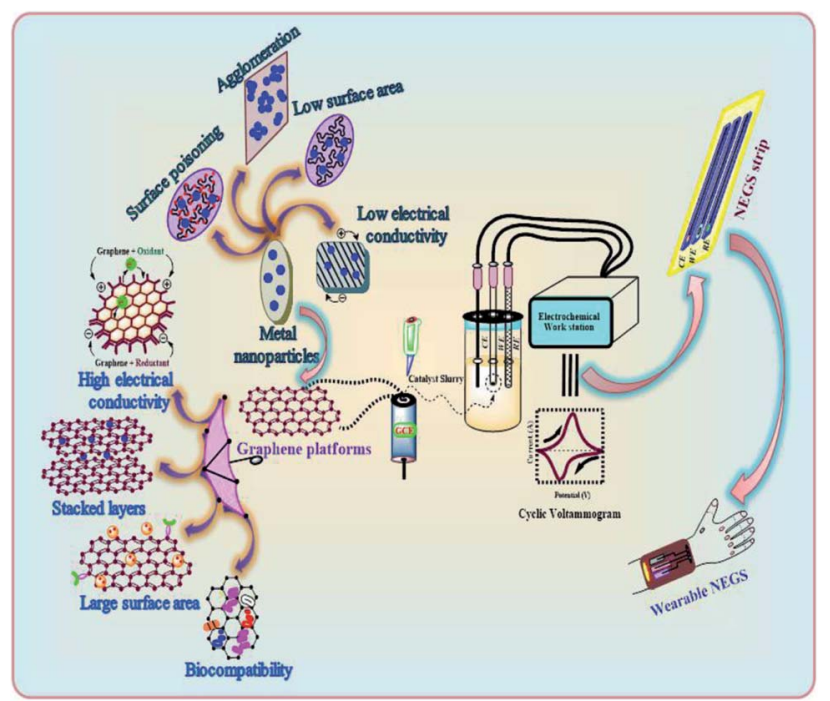

Fig. 2 Satisfaction of the requirements of NGES catalysts by graphene platforms and future developments.

Interestingly, heteroatom-doped graphene may effectively avoid the utilization of metal nanoparticles, which can drastically decrease the overall cost of a NEGS device. The satisfaction of the requirements of NEGS catalysts by graphene platforms and their future developments are depicted in Fig. 2.

\section{Timeline/progress of graphene materials in electrochemical sensors}

Despite its recent discovery, graphene has been effectively brought into the spotlight for the development of novel electrochemical sensors owing to its unique electrochemical and structural properties. The history of graphene began in 2004, with its discovery by Andre Geim and Konstantin Novoselov, ${ }^{59}$ and the unique features of graphene were brought into the limelight with the Nobel Prize in 2010.60 The journey of graphene in the field of sensors began in 2007, when Schedin et al. reported graphene-based gas sensors for individual gas molecules, including ammonia $\left(\mathrm{NH}_{3}\right)$, nitrogen dioxide $\left(\mathrm{NO}_{2}\right)$, carbon monoxide $(\mathrm{CO})$, and water $\left(\mathrm{H}_{2} \mathrm{O}\right) .{ }^{61}$ Extensive attention to graphene in electrochemical sensors was illuminated by the research efforts of Shan et al., who first described a graphenebased EGS in 2009. ${ }^{62}$ The constraints experienced in graphene synthesis were effectively addressed with the development of $\mathrm{rGO}$, and Zhou et al. exploited the advantages of rGO sheets in EGS in 2009. ${ }^{63}$ Extensive studies on graphene platforms in glucose sensors commenced after the accreditation of graphene research with the Nobel Prize in $2010 .^{64}$ The significant electrochemical responses of graphene-based EGS were further improved with nitrogen doping, which effectively eliminated the limitations of using metal nanoparticles. ${ }^{65}$ In the same year, metal nanoparticles were incorporated in a graphene-based film comprising gold (Au) nanoparticles/chitosan (CS); this was exploited as an effectual electrochemical probe in an EGS. ${ }^{66}$ Later, immense development efforts were exerted toward the fabrication of NEGS to address the significant constraints of EGS. Gao et al. developed the first graphene-based NEGS using graphene/platinum (Pt)-nickel (Ni) nanoparticles with superior durability. ${ }^{67}$ In 2012 , various research efforts were triggered to identify the influences of the morphology, size and structure of graphene and its anchored metal catalysts toward NEGS performance; 3D graphene has received much attention in this regard. ${ }^{68}$ In the preceding year, the significant limitations of precious metal nanoparticles were overcome by complexing non-precious metals and their oxides and conductive polymers with graphene nanosheets. In addition, the tremendous achievements in doping heteroatoms in graphene nanosheets encouraged its utilization in NEGS. The antifouling properties of the fabricated electrodes were addressed by intercalating the CS polymer with $\mathrm{rGO} / \mathrm{Ni}$ composite, which provided novel insights beyond the active sites and the electrical conductivity of the prepared catalysts for NEGS applications. ${ }^{69}$ Also, the anchoring of metal nanoparticles on nitrogen-doped graphene sheets was achieved during this period. In the past two years, immense research activities on the modification of $2 \mathrm{D}$ graphene into 3D structures have been triggered to increase the surface area and promote the interfacial contact between the current collector and the analyte, which may be beneficial for the adsorption of analytes and electron transfer. During this period, GO nanoribbons $\mathrm{s}^{70}$ and porous graphene foam along with metal oxide electrocatalysts ${ }^{71}$ have also been developed for enhanced NEGS performance. Overall, the trends and research interest in graphene-based catalysts in the development of NEGS have been progressively improved by advances in engineering, materials science, electrochemistry, nanoscience and technology, biomedical applications, etc.

\section{Research efforts}

A number of advancements have been achieved in graphenebased platform-equipped NEGS to increase the sensitivity and other significant electrochemical properties of sensors. The research efforts devoted to graphene-based platforms in NEGS have been segmented into six categories, and the significant efforts made in the above platforms are discussed in sequential order along with the portfolio of future progress.

\subsection{Precious metal nanostructures/graphene composites}

The technological advancements of noble metal nanostructures in a number of fields, owing to their intriguing size-dependent chemical, electrical, magnetic and optical properties in addition to their relatively easier synthesis and functionalization processes, have encouraged their utilization in NEGS. In addition, the ability of adopting multiple oxidation states and the formation of intermediates with the adsorbed/absorbed species and their easier activation for electrochemical reactions have further increased their utilization in NEGS. Furthermore, they can be easily integrated with the active graphene material without any alteration of its morphology and structure; the limitations exhibited by precious metal nanostructures were effectively addressed by the formation of composites with 
graphene. Pioneering work on noble metal nanomaterial/ graphene composites for NEGS applications was achieved by Kong et al., in which gold ( $\mathrm{Au}$ ) nanoparticles-decorated thionine (thi)-functionalized GO (Au/thi/GO) was prepared via a selfassembly technique and Au nanoparticles were densely anchored over the GO sheets with the aid of thionine. The electrocatalytic activity of $\mathrm{Au} /$ thi/GO was probed by $\mathrm{CV}$, and two anodic peaks were recognized at -0.34 and $0.23 \mathrm{~V} v s$. SCE under alkaline conditions; these were attributed to the generation of gluconolactone and its further oxidation, respectively. The detection limit of the $\mathrm{Au} /$ thi/GO/glassy carbon electrode (GCE) was found to be $0.05 \mu \mathrm{M}$ as evidenced by linear sweep voltammetry, owing to the improved electrocatalytic activity achieved via the synergistic effects of the three components. ${ }^{72}$ Liu et al. exploited the covalent interactions between bovine serum albumin (BSA)-protein-decorated rGO and Au nanoparticles, in which $80 \mathrm{~nm}$-sized $\mathrm{Au}$ nanoparticles were homogeneously anchored over the BSA/rGO surface. Facile electron-transfer kinetics toward glucose oxidation were stimulated at the $\mathrm{Au}$ / $\mathrm{BSA} / \mathrm{rGO}$ composite at the active sites of $\mathrm{Au}(\mathrm{OH})$ under alkaline conditions owing to its good conductivity and elevated electroactive surface area, as evidenced by the low charge transfer resistance $\left(R_{\mathrm{ct}}\right)$. The constructed $\mathrm{Au} / \mathrm{BSA} / \mathrm{rGO} / \mathrm{GCE}$ exhibited considerable NEGS performance at 0.0 vs. $\mathrm{Ag} / \mathrm{AgCl}$, and the practical reliability of these nanocomposites was verified in human blood serum with a relative standard deviation (RSD) of $3.5 \% .^{73} \mathrm{~A}$ combination of palladium (Pd) nanoparticles-supported GO (Pd/GO) was prepared using an ultrasonic technique, and the average diameter of the monodispersed Pd nanoparticles on GO sheets was found to be $4.25 \mathrm{~nm}$. The amperometric results depicted rapid NEGS performance via the $\mathrm{Pd}-\mathrm{OH}$ active sites under alkaline conditions at $0.4 v s$. SCE. ${ }^{74} \mathrm{Wu}$ et al. assembled Au/graphene and $\mathrm{Au} /$ CNTs composites using a supercritical $\mathrm{CO}_{2}$ fluid $\left(\mathrm{SCCO}_{2}\right)$, and the sensitivity of the prepared composites was enhanced by coupling with butylmethylpyrrolidinium bis(tri-fluoro methyl sulfonyl)imide (BMP-TFSI) ionic liquid (IL).

Systematic characterization revealed that the nanoparticles were decorated over both the graphene and CNTs with a mean diameter of $10 \mathrm{~nm}$, and the substantial obtained morphology was credited to the lower viscosity, gas-like diffusivity, and good $\mathrm{SCCO}_{2}$ penetration ability, which facilitated the transportation of the precursor all through the samples. Without the incorporation of BMP-TFSI, Au/CNTs exhibited improved electrooxidation in comparison with Au/graphene. Upon the incorporation of BMPTFSI, Au/graphene exhibited a pronounced sensing performance toward glucose (Fig. 3a) in comparison with Au/CNTs (Fig. 3b) owing to the influence of the IL on the suitability of the active graphene support (Fig. 3). The IL/SCCO $/ \mathrm{Au} /$ graphene/ GC disk exhibited superior electrochemical response over the $\mathrm{Au} /$ graphene composite owing to the homogeneous distribution of $\mathrm{Au}$ nanoparticles over the graphene support, achieved via $\mathrm{SCCO}_{2}$. The observed remarkable performance was attributed to the inordinate structural stability, reduced fouling of the absorbed intermediates, and suppression of graphene restacking via the formation of a functionalized layer on the graphene surface. Furthermore, the wrapping of graphene nanosheets with BMP-
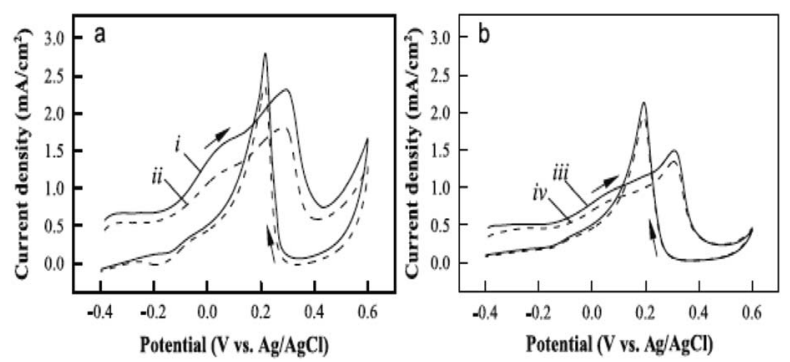

Fig. $3 \mathrm{CV}$ responses of (a) IL/SCCO $\mathrm{SCO}_{2}-\mathrm{Au} /$ graphene (curve (i)), IL/airAu/graphene (curve (ii)), (b) IL/SCCO $2-\mathrm{Au} / \mathrm{CNT}$ (curve (iii)), and IL/air$\mathrm{Au} / \mathrm{CNT}$ (curve (iv)) electrodes investigated in $5 \mathrm{mM}$ glucose solution at a scan rate of $10 \mathrm{mV} \mathrm{s}^{-1}$ (reproduced with permission from Elsevier; Wu et al. 2013).

TFSI overcame the $z$-direction conduction, which improved the electrochemical properties. ${ }^{75}$

$\mathrm{Wu}$ et al. described Pt nanoflowers-anchored graphene sheets (Pt/graphene) using a prototype-free approach; the average diameter of the prepared Pt nanoflowers, was found to be about $30 \mathrm{~nm}$, which were composed of $4 \mathrm{~nm}$-sized spherical nanoparticles. The electrocatalytic activity of $\mathrm{Pt} / \mathrm{graphene}$ was investigated using CV under neutral conditions, revealing three anodic peaks at $-0.4,0.09$ and $0.28 \mathrm{~V} v s$. SCE under the positive scan; these represent the electrochemical adsorption of glucose and the dehydrogenation of anomeric carbon, the electrooxidation of glucose into gluconic acid with the release of protons and further oxidation of glucose and its intermediates for the formation of gluconolactone/gluconic acid, respectively. The high tolerance of Pt/graphene/GCE toward chloride ions in a physiological environment was confirmed by the unchanged redox peak positions for the oxidation of glucose. The synergistic effects of the GO and Pt nanostructures facilitated a large surface area and edge-plane-like defects, which promoted the catalytic activity of the composite toward glucose detection. The glucose quantification studies in real samples obtained from a hospital exhibited a RSD of 7.6\%, indicating the excellent and reproducible performance of the aforementioned sensor in glucose detection. ${ }^{76}$ Au/graphene nanoribbon (GONR) composite was prepared using chemical and thermal deposition techniques, where the unzipping of MWCNTs provided GONR with an enhanced length to width ratio and a structure enriched with oxygen functionalities along with straight edges. The Au nanoparticles were homogenously aggregated at the edges of GONR; this was attributed to the existence of oxygenlabile functionalities at the edge planes of GONR, which attract the Au nanoparticles through 3D-specific non-covalent interactions. The electrooxidation peak potential of glucose observed at the $\mathrm{Au} / \mathrm{GONR} /$ carbon sheets (CS) was negatively shifted to $\sim 30 \mathrm{mV}$ in comparison with $\mathrm{Au} / \mathrm{CS}$ and Au/GONR/CS and provided an increased current density of $121.22 \%$, demonstrating the dominant effects of GONR in determining the reaction rate by increasing the adsorption kinetics. The incorporation of GONR enhanced the active surface area to $34.84 \%$ in $\mathrm{Au} / \mathrm{GONR} / \mathrm{CS}$, which increased the glucose oxidation peak current density to $200 \%$. Chloride ions are considered to 
be significant inhibiting species for Au-based NEGS because they dissolve in chloride ions instead of forming an oxide layer (Fig. 4b). This was effectively addressed with a permselective coating with a $\sim 3310 \mathrm{~nm}$ polypyrrole (PPy)/Nafion membrane (Fig. 4a). Although the electrooxidation response of the PPy/ Nafion membrane-coated Au/GONR/CS decreased, owing to the retardation of transportation of glucose to the electroactive sites, the selectivity of the fabricated sensors was greatly improved (Fig. 4c). The PPy/Nafion membrane significantly decreased the poisoning of $\mathrm{Au}$ nanoparticles from AA, $p$-acetamidophenol, and UA interference via charge repulsion processes and exhibited a linear increase with increasing glucose concentration (Fig. $4 \mathrm{~d}) .^{70}$

Ruiyi et al. prepared an $\mathrm{Au} / \mathrm{graphene}$ aerogel (Au/GA) by exploiting a chemical reduction technique, and the hierarchical features revealed that the prepared $\mathrm{Au} / \mathrm{GA}$ composite possessed a well-defined, well-oriented and $3 \mathrm{D}$ porous structure. The $\mathrm{CV}$ profile of the fabricated Au/GA/GCE exhibited glucose redox peaks at $0.078 / 0.104 \mathrm{~V}$ vs. $\mathrm{Ag} / \mathrm{AgCl}$ under alkaline conditions, and the obtained peak potential and oxidation peak current $\left(I_{\mathrm{pa}}\right)$ were lower and higher, respectively, in comparison with bare GCE, which demonstrated the excellent electrocatalytic activity of the prepared composite. Au/GA/GCE exhibited rapid NEGS performance owing to the well-defined 3D and oriented porous structure of GA, which facilitated glucose adsorption followed by its electrochemical oxidation. The reliability of Au/GA/GCE was validated by real sample analysis of glucose in human blood serum, with a recovery rate of $95.7 \%$ to $104.2 \% .{ }^{77}$ Joshi et al. prepared a nanocomposite comprising $50 \pm 5 \mathrm{~nm}$-sized silver (Ag) nanoparticles and GO (Ag/GO) using an electrodeposition technique. The voltammograms obtained under alkaline conditions revealed four distinct anodic peak potentials, representing the formation of $\mathrm{Ag}(\mathrm{OH})_{2}, \mathrm{Ag}_{2} \mathrm{O}$ monolayer, and $\mathrm{Ag}_{2} \mathrm{O}$ multilayer and the further reoxidation of $\mathrm{Ag}_{2} \mathrm{O}$ to $\mathrm{AgO}$, respectively. The obtained voltammograms confirmed that the analyte glucose was electrochemically oxidized at $\mathrm{AgO}$. The response of the $\mathrm{Ag} / \mathrm{GO}$ nanocomposites
(A)
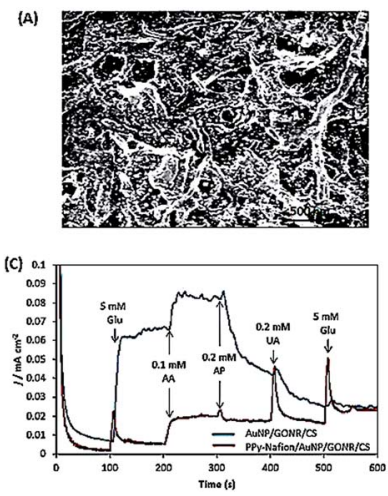
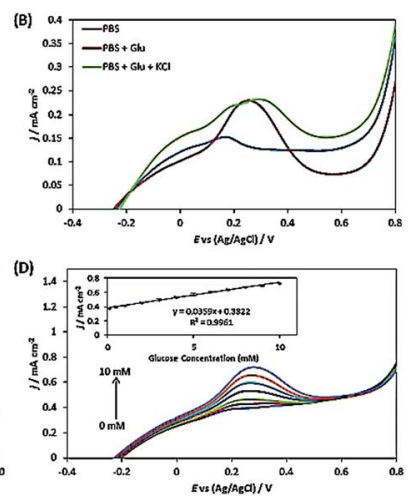

Fig. 4 (A) SEM image of PPy/Nafion membrane-coated Au/GONR/CS, (B) LSVsPPy/Nafion membrane-coated Au/GONR/CS in 0.1 M PBS (pH $=7.0$ ) involving $5 \mathrm{mM}$ glucose in the presence and absence of $0.1 \mathrm{M}$ $\mathrm{KCl}$ at a scan rate of $10 \mathrm{mV} \mathrm{s}^{-1}$, (C) chronoamperometry responses of Au/GONR/CS and PPy/Nafion membrane-coated Au/GONR/CS upon the injection of various interfering species and (D) LSVs with different concentrations of glucose on a PPy/Nafion/AuNP/GONR/CS electrode (reproduced with permission from Elsevier; Ismail et al. 2014). was extended to human blood analysis, showing substantial electrochemical detection of glucose. ${ }^{78} \mathrm{Hoa}$ et al. fabricated NEGS with an amalgam of $\mathrm{Pt} / \mathrm{GO}$ hydrogel $(\mathrm{Pt} / \mathrm{GOH})$ and evaluated its electrochemical performance under alkaline conditions. The morphological images revealed that $6 \mathrm{~nm}$-sized Pt nanoparticles were uniformly anchored on the GO surface. Under alkaline conditions, $\mathrm{Pt}^{0}$ adsorbed $\mathrm{OH}^{-}$ions and was converted to $\mathrm{Pt}(\mathrm{II})$, which underwent further electrochemical oxidation, yielding $\mathrm{Pt}(\mathrm{III})$; the analyte glucose was electrochemically oxidized at the $\mathrm{Pt}($ III) active centres. The optimized Pt content in the Pt/GOH composite was found to be $15 \mathrm{mg}$, and a further increment in Pt content lead to agglomeration, which decreased the electrochemical performance. The Pt/GOH NEGS exhibited excellent performance toward electrochemical glucose oxidation at $0.1 \mathrm{~V} v$ s. $\mathrm{Ag} / \mathrm{AgCl}$. Interestingly, real-time applications were achieved with horse and rabbit serums at Pt/GOH/GCE; the superior obtained electrochemical performance is ascribed to the $3 \mathrm{D}$ porous structure and homogeneous active sites of the $\mathrm{Pt} / \mathrm{GOH}$ composite. ${ }^{79}$ Shu et al. described 3D nanocomposites comprising $\mathrm{Au}$ nanoparticles-anchored graphene sheets (Au/graphene) using an electrochemical approach wherein $\mathrm{Au}^{+}$ions and GO sheets were simultaneously reduced. The CV results indicated that the electrooxidation of glucose at Au/graphene/GCE was achieved at $\sim 0 \mathrm{~V}$ vs. $\mathrm{Ag} / \mathrm{AgCl}$, while the formation of gluconolactone was occurred via a two-electron-transfer reaction. For the effectual amperometric sensing, the applied potential was optimized with a series of experiments, and it was concluded that an applied potential of $0 \mathrm{~V}$ vs. $\mathrm{Ag} / \mathrm{AgCl}$ provided a concordant increase of oxidation current with increasing glucose concentration with stable signals (Fig. 5a). The accumulation of intermediates observed over the electrode surface with other applied potentials decreased the sensitivity of the constructed sensors (Fig. 5a). The as-fabricated $\mathrm{Au} /$ graphene/GCE exhibited excellent selectivity toward glucose against interfering species (Fig. 5b) and provided good amperometric response toward glucose oxidation under neutral conditions (Fig. 5c and d), and its analytical reliability was validated with real sample analysis of glucose in mice blood serum with an RSD of $0.36 \%$. The 3D framework of graphene protected the $\mathrm{Au} /$ graphene nanocomposites from deformation, agglomeration, and disintegration under the severe electrochemical conditions. The existence of large pores in the 3D Au/graphene nanocomposites ensured maximum glucose adsorption, which facilitated the diffusion of glucose via the electrolyte/electrode interface, enabling maximum contact with a number of active surfaces. ${ }^{80}$ A highly electroactive Au/SWCNT/rGO electrocatalyst was prepared using bovine serum albumin (BSA) as a reducing agent; the $\pi-\pi$ stacking interaction exerted between the SWCNTs and rGO sheets induced the homogeneous distribution of SWCNTs over the rGO sheets, in which the Au nanoparticles were densely anchored. The as-fabricated Au/SWCNT/rGO/GCE effectively detected glucose under alkaline conditions. The incorporation of hierarchical tortuous SWCNTs in GO sheets not only prevented the rGO sheets from restacking but also served as nanowires to facilitate the electron transfer. ${ }^{81}$ Thanh et al. exploited the CVD technique for the preparation of $\mathrm{Au}$ nanoparticles-encapsulated few-layered graphene (FLG) nanohybrids on indium tin oxide (ITO) (Au/FLG/ITO). The grain size of 

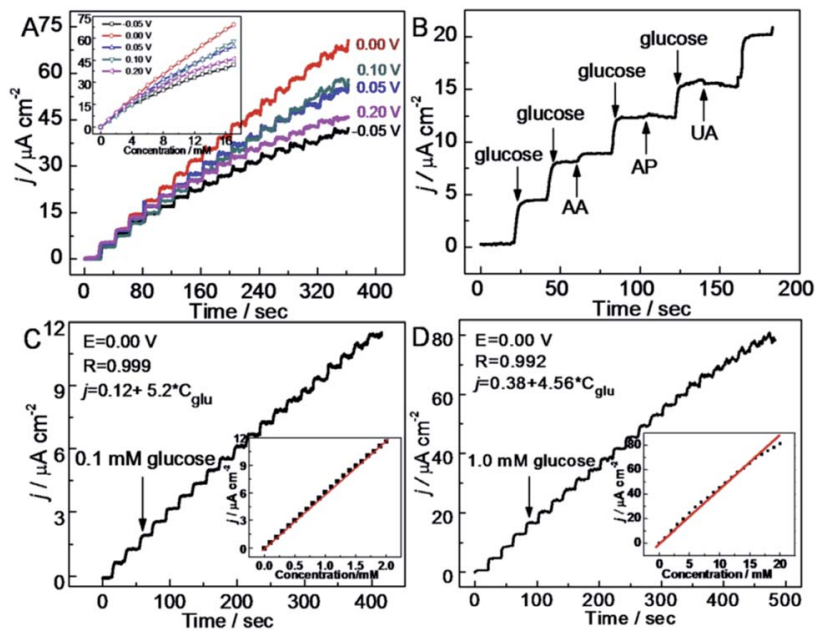

Fig. 5 (A) Amperometric responses of Au/graphene/GCE in 0.1 M PBS $(\mathrm{pH} 7.4)$ with a continual addition of $1.0 \mathrm{mM}$ glucose as a function of potential (inset: corresponding calibration plots), (B) chronoamperometry responses of Au/graphene/GCE with consecutive additions of $1.0 \mathrm{mM}$ glucose, $0.1 \mathrm{mM} \mathrm{AA}, 0.05 \mathrm{mM} \mathrm{AP}$ and $0.05 \mathrm{mM} \mathrm{UA}$ at an applied potential $0.0 \mathrm{~V}$ vs. $\mathrm{Ag} / \mathrm{AgCl}$, and (C and D) chronoamperometry responses of Au/graphene/GCE with consecutive additions of (C) $0.1 \mathrm{mM}$ and (D) $1.0 \mathrm{mM}$ glucose (inset: calibration curve for the amperometric $i-t$ curve) (reproduced with permission from Elsevier; Shu et al. 2015).

the Au nanoparticles anchored over the graphene matrix was purely dependent upon the electroless deposition time, and the electrodeposition time of 3 min showed increased electrocatalytic glucose oxidation owing to the uniform size of the $15 \mathrm{~nm} \mathrm{Au}$ nanoparticles and their superior interaction with FLG sheets over 1 and $5 \mathrm{~min}$ of electrodeposition. Au/FLG/ITO exhibited the maximum electrooxidation, as evidenced from the higher $I_{\mathrm{pa}}$ of $\sim 583 \mu \mathrm{A}$ and the lower peak to peak separation $\left(\Delta E_{\mathrm{p}}\right)$ of $\sim 394 \mathrm{mV}$ in comparison with Au/ITO under neutral conditions. The facile electron transfer kinetics observed at the Au/FLG nanohybrids is purely attributed to the uniform particle size of the Au nanoparticles and their strong interactions with the graphene sheets. ${ }^{82}$

\subsection{Non-precious metal nanostructures/graphene composites}

Although the electrochemical properties of NEGS can be improved by devoting research efforts to precious metal nanoparticles/graphene composites, the large scale applications of precious metal nanoparticles are largely hindered by their limited availability, high cost, chloride poisoning, surface poisoning/fouling from adsorbed intermediates, etc. This can be effectively addressed with non-precious metal nanoparticles/ graphene composites, and the progress made on the above is sequentially described as follows: the spin coating technique was exploited for coating $\mathrm{Cu}$ nanowires on graphene transparent electrode (GTE) (Cu/GTE). The preparation of GTE was achieved by the incorporation of GO on cellulose acetate microfiltration membranes followed by pressing on polyethylene terephthalate (PET) sheets, in which $180 \mathrm{~nm}$-diameter $\mathrm{Cu}$ nanowires were homogeneously deposited via a spin coating technique. The electrocatalytic activity of the fabricated sensors was investigated using CV in neutral medium, and effectual electrooxidation of glucose was achieved via the $\mathrm{CuOOH} / \mathrm{Cu}(\mathrm{OH})_{4}$ active species. The effective combination of $\mathrm{Cu}$ nanowires and GTE showed effectual amperometric glucose oxidation owing to direct electron transfer between the $\mathrm{Cu}$ nanowires and GTE. The fabricated $\mathrm{Cu} / \mathrm{GTE}$ provided improved performance toward real blood glucose sample analysis in human blood serum with reliable glucose detection. The newly developed GTE evinced a number of advantages, such as low resistance, disposability, a wide range window, good transparency, low cost and chemical stability, increasing its viable application. ${ }^{83}$

Wang et al. developed NEGS with an urchin-like Ni nanostructures/graphene composite by a simple in situ chemical reduction technique. From morphological studies, it is clear that urchin-like $\mathrm{Ni}$ nanostructures were effectively embedded on the GN matrix, wherein the spherical Ni core bears various spear-like branches with a diameter of $200 \mathrm{~nm}$. $\mathrm{Ni}($ III) species generated in alkaline medium electrooxidized glucose and provided good amperometric responses at $0.5 v s$. SCE. ${ }^{84}$ Wang et al. reported an electrophoretic deposition (EPD) technique for the preparation of $\mathrm{Cu} / \mathrm{rGO}$ composite-based thin films over the Au interface, and the influence of deposition time was investigated in detail. The morphological images revealed that $\mathrm{Cu}$ nanoparticles with a mean diameter of $67 \pm 6 \mathrm{~nm}$ were anchored on the rGO surface with a deposition time of $2 \mathrm{~min}$. The CV behaviour of the $\mathrm{Cu} / \mathrm{rGO}$ hybrid under alkaline conditions exhibited significant increases in the anodic peaks at -0.35 and $-0.312 \mathrm{~V} v s$. $\mathrm{Ag} / \mathrm{AgCl}$ and in the cathodic peaks at -0.38 and $-0.80 \mathrm{~V} v s$. $\mathrm{Ag} / \mathrm{AgCl}$ upon the addition of glucose. The as-developed $\mathrm{Cu} / \mathrm{rGO} / \mathrm{Au}$ platform exhibited considerable amperometric performance at $0.55 \mathrm{vs}$. $\mathrm{Ag} / \mathrm{AgCl}$. This competitive approach to conventional electrochemical probe processes has advantages, such as a high metal nanoparticle deposition rate, thickness controllability and good uniformity. The practical applicability of the fabricated sensor was extended to real sample analysis in human blood serum for the detection of glucose concentration with an appreciable recovery. ${ }^{85}$ Shen et al. developed a Ni nanoparticles/attapulgite (ATP)/rGO composite via an electrochemical co-deposition technique, and the hierarchical features revealed that the inhibition of ATP favoured the active surface for the uniform dispersion of Ni nanoparticles. The $\mathrm{CV}$ behaviour of Ni/ATP/rGO/GCE depicted that the active $\mathrm{Ni}$ (III) species electrooxidized glucose into gluconolactone, and the amperometric results depicted the superior amperometric performance of $\mathrm{Ni} / \mathrm{ATP} / \mathrm{rGO} / \mathrm{GCE}$ at $0.5 v s$. $\mathrm{Ag} /$ $\mathrm{AgCl}$. The natural nanostructured ATP-bearing zeolite-like channels and their conjugation with rGO sheets favoured a large specific surface area, high absorption capacity, biocompatibility, and increased absorbability, which collectively improved the performance of the NEGS. Glucose quantification in real sample analysis, i.e., human serum, revealed excellent analyte recovery. ${ }^{86}$ Tehrani et al. taped Kapton tape (polyimide) over a polyvinyl chloride transparent substrate and subjected the system to laser engraving. Direct laser-engraved graphene electrodes (DLEG) were fabricated with the designed pattern using graphic software (Fig. 6), in which the $\mathrm{Cu}$ nanocubes were 
homogeneously loaded via the pulse electrodeposition technique. The topological features revealed the appearance of thin interconnected nanosheets of graphene and uniform, highdensity deposition of $\mathrm{Cu}$ nanocubes with a mean diameter of less than $30 \mathrm{~nm}$. In comparison with DLEG, Cu/DLEG exhibited an increased charge transfer rate, as substantiated from the lower charge resistance of $R_{\mathrm{ct}}=21 \Omega$. The $\mathrm{Cu}(\mathrm{III})$ species generated from Cu/DLEG under alkaline conditions electrooxidized glucose into hydrolyzate gluconic acid and exhibited a rapid response time, low detection limit, broad linear range and elevated sensitivity at $0.55 \mathrm{~V} v$ s. $\mathrm{Ag} / \mathrm{AgCl}$. Furthermore, $\mathrm{Cu}$ / DLEG effectively detected glucose in real samples, including tears, saliva, sweat, and urine, with good stability. ${ }^{88}$ An exfoliated GO sheet formulation was cast in a polytetrafluoroethylene (PTFE) mold by Wang et al.; the GO paper peeled off from a PTFE mold exhibited a layer-by-layer structure with a film thickness of $10 \mu \mathrm{m}$. The obtained GO paper was chemically reduced to $\mathrm{rGO}$, and $\mathrm{Cu}$ nanoflowers were electrochemically deposited on the rGO paper. The morphological properties of the $\mathrm{Cu}$ nanostructures were purely dependent upon the electrodeposition time, and the nanocubes formed in $30 \mathrm{~s}$ gradually grew into flower shapes at $120 \mathrm{~s}$; after that, no significant morphological changes were observed. No significant current variation was observed upon bending the $\mathrm{Cu} / \mathrm{rGO}$ paper at $180{ }^{\circ} \mathrm{C}$, which demonstrated the extraordinary flexibility and physical stability of the as-prepared paper electrode. The reliability of the $\mathrm{Cu} / \mathrm{rGO}$ paper was verified by real sample analysis of glucose in human serum, which exhibited a RSD of $<3 \%$; this reveals the significance of the aforementioned sensor in glucose quantification. ${ }^{87}$ The significant electrochemical performance obtained on precious/non-precious metal nanoparticles/ graphene-based NEGS is depicted in Table $1 .^{\mathbf{7 0 , 7 2 - 8 9}}$

\subsection{Non-precious metal oxide nanostructures/graphene composites}

Although the utilization of non-precious metal nanoparticles/ graphene composites eventually decreases the overall cost of a glucose device, the stability of non-precious metal catalysts in neutral or low pH conditions is inferior owing to the high dependency of $\mathrm{MOOH}$ species for the electrooxidation of glucose; this has increased the focus on non-precious metal oxide/graphene composites in NEGS.

3D porous freestanding graphene foam electrodes (PGF) decorated with copper hydroxide $\left(\mathrm{Cu}(\mathrm{OH})_{2}\right)$ porous nanorods

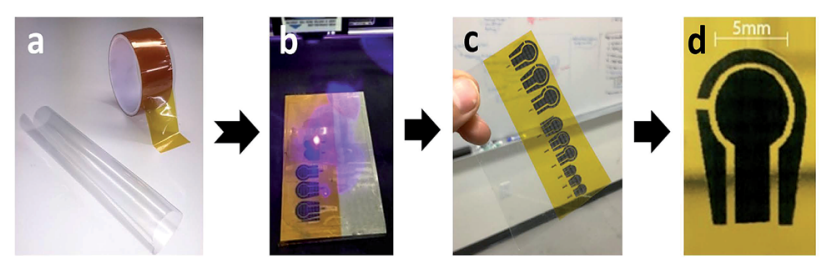

Fig. 6 Optical images of (a) taping Kapton tape on PVC, (b and c) direct laser reduction of Kapton tape for the formation of graphenebased sensor electrodes and (d) a DREG 3-electrode platform (reproduced with permission from Nature Scientific Reports; Tehrani et al. 2016).
$\left(\mathrm{Cu}(\mathrm{OH})_{2} / \mathrm{PGF}\right)$ were utilized as a sensing probe in NEGS, and the porous $3 \mathrm{D}$ interconnected $\mathrm{Ni}$ foam was utilized as a template for the growth of PGF. Owing to the thermal expansion coefficient differences between $\mathrm{Ni}$ foam and graphene, ripples and wrinkles were formed over the surface of the graphene film (Fig. 7a-c). The morphological images clearly showed that 100 to $400 \mathrm{~nm}$-sized flowerlike structured $\mathrm{Cu}(\mathrm{OH})_{2}$ comprising $100 \mathrm{~nm}$ nanorods were uniformly dispersed on the PGF (Fig. 7b). The uniform distribution of carbon, oxygen, and $\mathrm{Cu}$ atoms in $\mathrm{Cu}(\mathrm{OH})_{2} / \mathrm{PGF}$ was confirmed by elemental dispersive X-ray spectroscopy (EDAX) elemental mapping (Fig. 7d). $\mathrm{Cu}(\mathrm{OH})_{2} / \mathrm{PGF}$ exhibited a glucose oxidation potential peak at $0.55 \mathrm{~V}$ vs. $\mathrm{Ag} / \mathrm{AgCl}$, which was attributed to the conversion of glucose to gluconolactone by the electroactive species $\mathrm{CuOOH}$ under alkaline conditions. The prepared electrochemical probe exhibited good amperometric results in glucose quantification. The existence of an interconnected porous structure in PGF facilitated the loading of metal nanorods and extended the mass transfer by enabling the analyte to approach the active sites; also, the rippled, wrinkled functionalities of PGF increased the attachment of $\mathrm{Cu}(\mathrm{OH})_{2}$ through better mechanical and chemical interlocking. ${ }^{71}$

An electrochemical probe comprising graphene-wrapped cuprous oxide $\left(\mathrm{Cu}_{2} \mathrm{O}\right)$ nanocubes was achieved by a simple chemical reduction technique; the $\mathrm{Cu}_{2} \mathrm{O}$ nanostructures in the $\mathrm{Cu}_{2} \mathrm{O}$ /graphene composite existed as uniform cubic shapes, and their average edge length was found to be 207.6 to $142.7 \mathrm{~nm}$. The prime sensing applications of the graphene-wrapped $\mathrm{Cu}_{2} \mathrm{O}$ nanocubes were examined using amperometric studies, which revealed that $0.6 \mathrm{~V} v s$. $\mathrm{Ag} / \mathrm{AgCl}$ was the optimum potential for effectual electrooxidation, and the fabricated $\mathrm{Cu}_{2} \mathrm{O} /$ graphene/ GCE exhibited an excellent amperometric performance in alkaline conditions. Furthermore, the as-developed catalyst exhibited high chloride tolerance and robust electrochemical stability, which significantly increased the electron transfer during the electrochemical reaction..$^{90}$ The implementation of a green reducing agent for the preparation of copper oxide $(\mathrm{CuO}) / \mathrm{rGO}$ composite $(\mathrm{CuO} / \mathrm{rGO})$ was accomplished with the aid of isopropanol, which served as both solvent and reducing regent. In the preparation of the $\mathrm{CuO} / \mathrm{rGO}$ composite, the weak $\mathrm{H}$-bonds between GO and isopropanol were broken by the inclusion of $\mathrm{Cu}^{2+}$ ions, which electrostatically interacted with the oxygen-labile functionalities of GO. This was followed by hydrolysis, which led to the nucleation and growth of $\mathrm{CuO}$ crystallites, enabling the formation of $\mathrm{CuO}$ nanorods; the simultaneous reduction of $\mathrm{Cu}^{2+}$ ions and GO led to the formation of $\mathrm{CuO} / \mathrm{rGO}$ nanocomposites. The $\mathrm{CuO}$ nanostructures prepared via an in situ approach on the rGO sheets exhibited rod-like nanoparticles with an average length of 20 to $30 \mathrm{~nm}$. CV studies revealed that the oxidation of glucose occurred at $0.4 \mathrm{~V}$ vs. SCE for $\mathrm{CuO} / \mathrm{rGO} / \mathrm{GCE}$; the electroactive $\mathrm{Cu}^{3+}$ species, $\mathrm{CuOOH}$ and $\mathrm{Cu}(\mathrm{OH})_{4}{ }^{-}$, electrooxidized the glucose into gluconolactone under alkaline conditions. The synergistic effects of the semiconducting properties of $\mathrm{CuO}$, with a band gap of $1.2 \mathrm{eV}$, and the highly conductive rGO sheets enhanced the amperometric glucose detection properties of the sensor. ${ }^{91}$ 


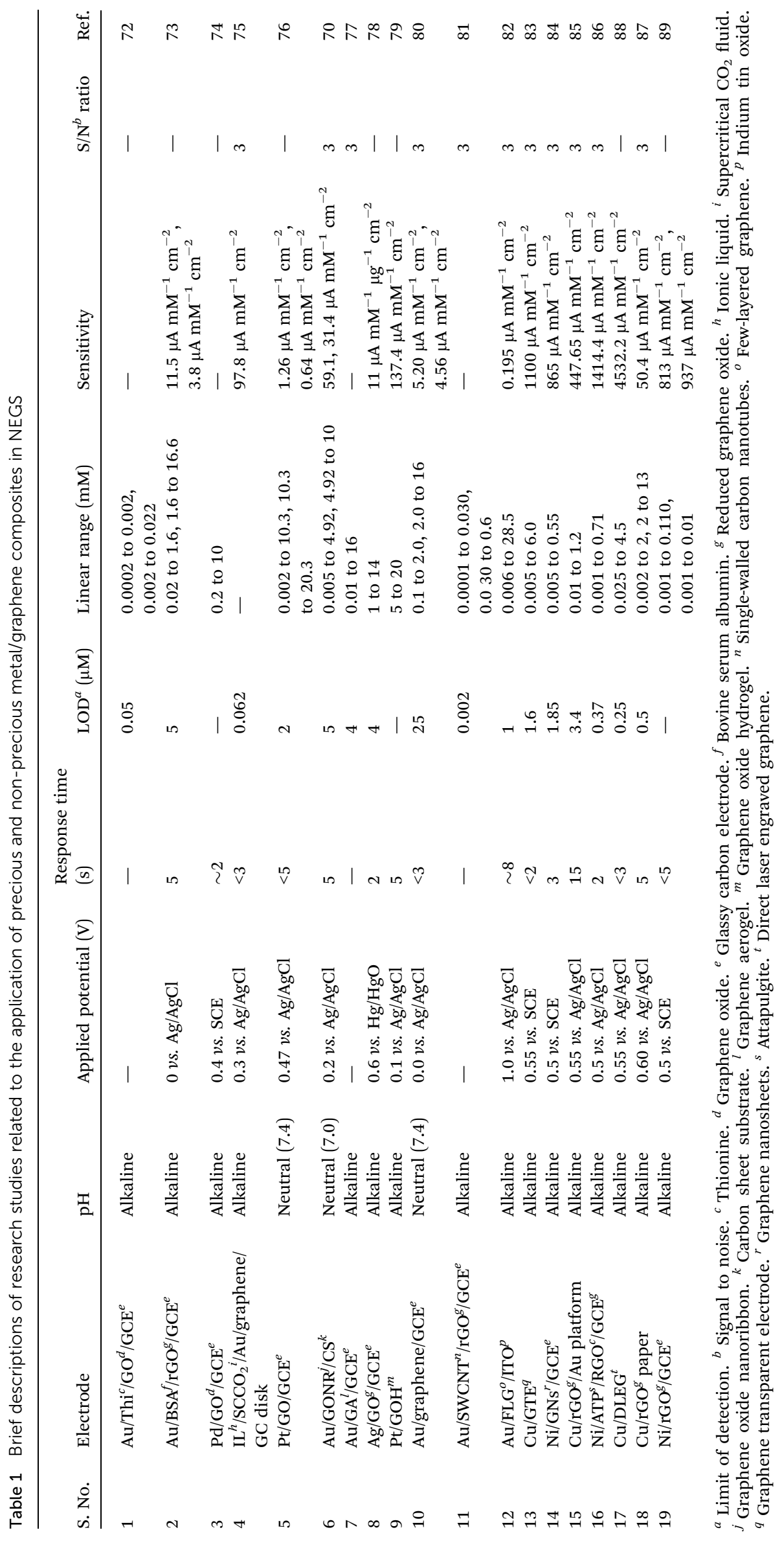


A combination of $\mathrm{CuO}$ nanoparticles with 3D porous $\mathrm{rGO}$ (PrGO) was achieved via a hydrothermal approach, and morphological studies revealed that the $\mathrm{CuO}$ nanoparticles were loaded on the wrinkled flakelike PrGO, in which $\mathrm{CuO} /$ PrGO exhibited adjacent fringe spacing of $0.27 \mathrm{~nm}$ in the direction of the wire growth. $\mathrm{CuO} / \mathrm{PrGO}$ exhibited a pronounced electrooxidation behavior toward glucose at $0.55 \mathrm{~V} v s . \mathrm{Ag} / \mathrm{AgCl}$, as evidenced from the maximum peak current of $95 \mu \mathrm{A}$; this is superior to that of $\mathrm{CuO} / \mathrm{rGO} / \mathrm{GCE}(67 \mu \mathrm{A})$ owing to the mesoporous structure of PrGO, which exposed the target molecules with a high utilization efficiency rate on the active sites of CuO. The fabricated $\mathrm{CuO} / \mathrm{PrGO} / \mathrm{GCE}$ exhibited considerable amperometric responses toward glucose detection in alkaline conditions owing to the 3D porous structure of PrGO, which facilitated a higher electron transfer rate at the active sites of CuO. This fabricated material provided improved performance toward real blood glucose sample analysis, i.e., analyzing glucose in human blood serum with a $4.4 \%$ RSD. ${ }^{92}$ Ye et al. reported the combination of $\mathrm{rGO}$, carbon nanofiber (CNF) and $\mathrm{CuO}$ nanoneedles $(\mathrm{CuO} / \mathrm{rGO} / \mathrm{CNF})$ as an excellent electrochemical probe for NEGS. The CNFs were dispersed among the rGO layers, in which the $\mathrm{CuO}$ flower-like aggregates were uniformly distributed. The presence of $1 \mathrm{D} \mathrm{CNF}$ and $2 \mathrm{D}$ rGO sheets maximized the porosity, electrical network and basal spacing, and the 1D CNF bridged defects for electron transfer between the $\mathrm{rGO}$ nanosheets. $\mathrm{CuO} / \mathrm{rGO} / \mathrm{CNF} / \mathrm{GCE}$ exhibited pronounced electrooxidation behavior toward glucose under a alkaline conditions at $0.6 \mathrm{~V} v s$. SCE, as evidenced from the fast response time, low detection limit, high sensitivity and broad linear range. The practical applicability of the fabricated sensor was extended to real sample analysis in human saliva for the detection of glucose, with an appreciable recovery of $99.7 \%$ to $101.3 \%{ }^{93}$

Zheng et al. acquired a $\mathrm{CuO} / \mathrm{rGO}$ composite via a facile microwave approach, and morphological studies revealed that the $\mathrm{CuO}$ nanostructures exhibited a porous $3 \mathrm{D}$ rambutan-like microstructure with an average size of $0.5 \mu \mathrm{m}$ and were effectively loaded over the wrinkled rGO sheets. Owing to the high surface area and elevated electron transfer rate of $\mathrm{rGO}, \mathrm{CuO} /$ $\mathrm{rGO} / \mathrm{GCE}$ exhibited higher electrooxidation of glucose than the individual components via $\mathrm{Cu}(\mathrm{III})$ under alkaline conditions. The practicability of $\mathrm{CuO} / \mathrm{rGO} / \mathrm{GCE}$ was also confirmed by its good recovery rate of $94.8 \%$ to $101.4 \%$ for human blood samples. ${ }^{94}$ Mei et al. assembled a uniform $\mathrm{Cu}_{2} \mathrm{O}$ nanoclusters/ rGO composite by a solvothermal technique using poly(vinyl pyrrolidone)-poly(methacrylamide)-poly(vinyl imidazole)tri block polymer and L-glutamic acid as a shape-directing and reducing agents, respectively. The morphological characterization revealed that the $\mathrm{Cu}_{2} \mathrm{O}$ nanoclusters had a narrow size distribution of 1.2 to $3.9 \mathrm{~nm}$, with a mean diameter of $2.6 \mathrm{~nm}$. $\mathrm{Cu}_{2} \mathrm{O} / \mathrm{rGO} / \mathrm{GCE}$ demonstrated considerable amperometric response toward glucose under alkaline conditions at $0.6 \mathrm{~V} v s$. SCE. $\mathrm{Cu}_{2} \mathrm{O} / \mathrm{rGO} / \mathrm{GCE}$ was not influenced by interference molecules and exhibited rapid results toward real blood glucose sample analysis. ${ }^{95}$ Feng et al. exploited a facile solvothermal technique for the preparation of $\mathrm{Cu}_{2} \mathrm{O}$ nanowire/rGO composites; the $\mathrm{Cu}_{2} \mathrm{O}$ existed as nanowires and was uniformly

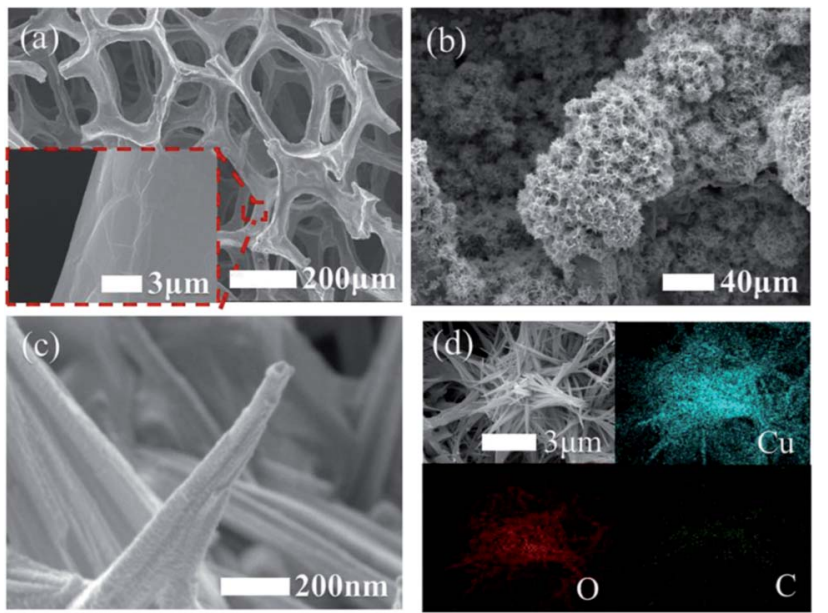

Fig. 7 Field emission SEM (FE-SEM) images of (a) PGF, (b) $\mathrm{Cu}(\mathrm{OH})_{2} /$ PGF and (c) $\mathrm{Cu}(\mathrm{OH})_{2}$ single nanorod; (d) FE-SEM image of $\mathrm{Cu}(\mathrm{OH})_{2} /$ PGF and energy dispersive spectroscopy (EDS) elemental mappings of copper, oxygen, and carbon atoms (reproduced with permission from Elsevier; Shackery et al. 2016).

distributed over the corrugated and crumpled rGO sheets, with a mean diameter of $5 \mathrm{~nm}$. The electrocatalytic activity of the fabricated $\mathrm{Cu}_{2} \mathrm{O} / \mathrm{rGO} / \mathrm{GCE}$ was investigated by $\mathrm{CV}$ in alkaline medium, and the defined steady state amperometric current response was observed at $0.55 \mathrm{~V} v s$. SCE within $2 \mathrm{~s}$ upon the addition of glucose. $\mathrm{Cu}_{2} \mathrm{O} / \mathrm{rGO} / \mathrm{GCE}$ exhibited a gradient of not less than $69.50 \mu \mathrm{AmM}^{-1}$ and a linear range of $10 \mu \mathrm{M}$ to $0.1 \mathrm{mM}$; also, it was free from the influence of interference molecules. ${ }^{96}$ A few additional research efforts have been devoted to $\mathrm{CuO} /$ graphene nanocomposites, and their significant NEGS performance is described in Table 2.97-106 Cobalt oxide $\left(\mathrm{Co}_{3} \mathrm{O}_{4}\right)$ nanoflowers-decorated $\mathrm{GO}$ hydrogel $(\mathrm{GOH}), \mathrm{Co}_{3} \mathrm{O}_{4} / \mathrm{GOH}$, was achieved by a hydrothermal technique; the aggregation of nanopetals over the 3D GO sheets led to the formation of $\mathrm{Co}_{3} \mathrm{O}_{4}$ nanoflowers, as evidenced from morphological studies. The CV behaviour of the fabricated $\mathrm{Co}_{3} \mathrm{O}_{4} / \mathrm{GOH} / \mathrm{GCE}$ exhibited a pronounced glucose oxidation peak at $0.7 \mathrm{~V} v s$. $\mathrm{Ag} / \mathrm{AgCl}$ and exhibited 20 fold enhanced sensitivity $\left(492.8 \mu \mathrm{A} \mathrm{mM} \mathrm{m}^{-1} \mathrm{~cm}^{-2}\right)$ over GOH $\left(24.06 \mu \mathrm{AmM}^{-1} \mathrm{~cm}^{-2}\right)$. On account of the effective charge transfer between $\mathrm{Co}_{3} \mathrm{O}_{4}$ and the conductive $\mathrm{GOH}$, the fabricated composite afforded a wide linear range of $0.25 \mathrm{mM}$ to $10 \mathrm{mM}$ and a response time of $8 \mathrm{~s}$. In comparison with the commercial ACCU-CHEK ${ }^{\circledR}$ Performa Blood Glucose Meter, $\mathrm{Co}_{3} \mathrm{O}_{4} / \mathrm{GOH} / \mathrm{GCE}$ exhibited concentration differences of $1.21 \%$ and $1.34 \%$ for horse and rabbit serum, respectively. ${ }^{107}$

$\mathrm{A} \mathrm{Co}_{3} \mathrm{O}_{4} / \mathrm{rGO}$ composite was prepared by a facile one-pot chemical method, and its hierarchical features revealed that the hybrid possessed evenly distributed $\mathrm{Co}_{3} \mathrm{O}_{4}$ nanoparticles over the curled graphene sheets with a mean particle size of 5 to $10 \mathrm{~nm}$. In addition, the influences of time and temperature on the unique properties of $\mathrm{Co}_{3} \mathrm{O}_{4} / \mathrm{rGO}$ were analysed; $\mathrm{Co}_{3} \mathrm{O}_{4} / \mathrm{rGO}$ prepared at $150{ }^{\circ} \mathrm{C}$ at $3 \mathrm{~h}$ offered superior electrocatalytic activity, owing to its smaller size, high rate of electrolyte transportation, uniform distribution and high surface area in comparison with other nanostructures. The $\mathrm{CV}$ behaviour of 


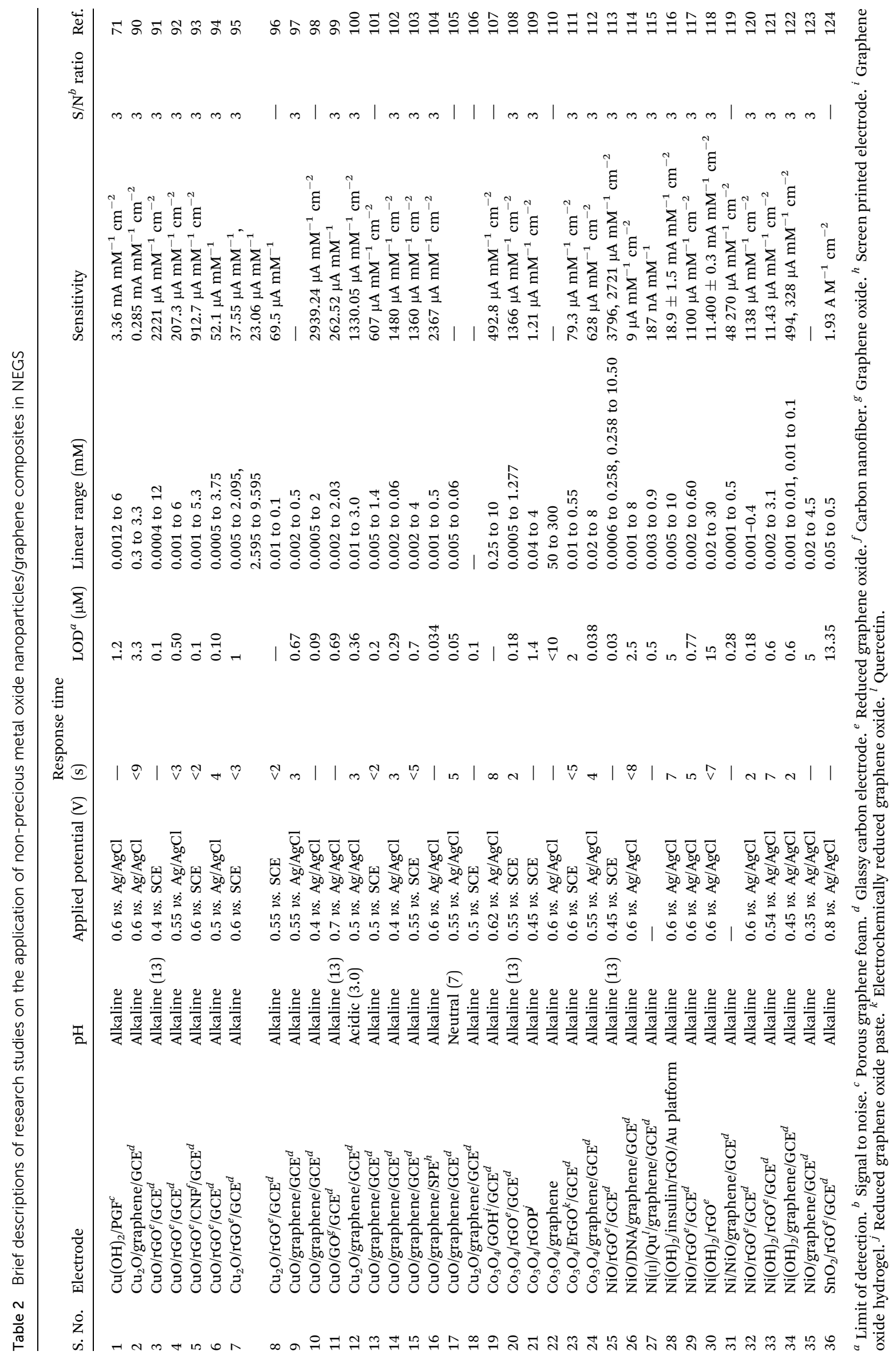




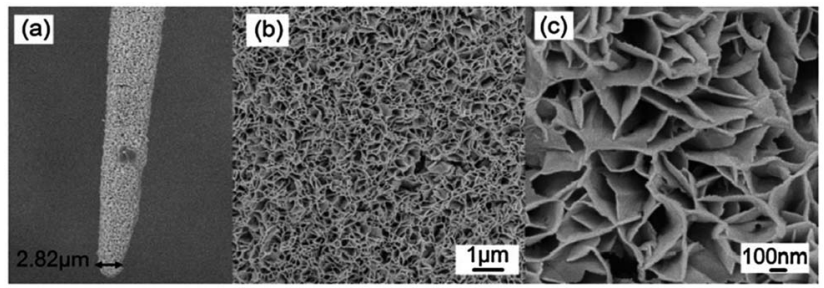

Fig. 8 (a-c) SEM images of a $\mathrm{CO}_{3} \mathrm{O}_{4} /$ graphene needle electrode with various magnifications (reproduced with permission from Royal Society of Chemistry; Wang et al. 2012).

$\mathrm{Co}_{3} \mathrm{O}_{4} / \mathrm{rGO} / \mathrm{GCE}$ exhibited a glucose oxidation peak at $0.55 \mathrm{~V} v$. SCE in alkaline medium and showed excellent amperometric behaviour toward glucose detection. The reliability of the fabricated electrode was verified by analyzing glucose in human blood serum, and the observed recovery rate was in the range of $102.85 \%$ to $104.10 \%$, which demonstrates its applicability. ${ }^{108}$ Heidari et al. electrodeposited $\mathrm{Co}_{3} \mathrm{O}_{4}$ nanoparticles on rGO paste (rGOP), and morphological studies clearly depicted the existence of a 3D, scaly network of graphene wherein 60 to 100 nm-sized $\mathrm{Co}_{3} \mathrm{O}_{4}$ nanoparticles were deposited onto the jagged network of graphene. The analytical performance of the fabricated $\mathrm{Co}_{3} \mathrm{O}_{4} / \mathrm{rGOP}$ depicted good amperometric response under alkaline conditions at $0.45 \mathrm{~V} v s$. SCE. The interference molecules, including saccharose, fructose, L-histidine, L-phenylalanine, and L-methionine, did not influence the NEGS performance of $\mathrm{Co}_{3} \mathrm{O}_{4} / \mathrm{rGOP}$; the practicability of $\mathrm{Co}_{3} \mathrm{O}_{4} / \mathrm{rGOP}$ was also extended to human blood analysis, showing a recovery of $97.2 \%$ to $103.4 \% .{ }^{109}$ Wang et al. gathered active graphene prepared by CVD over a glass micropipette and electrochemically deposited $\mathrm{Co}_{3} \mathrm{O}_{4}$ nanostructures at the micropipette tip $\left(\mathrm{Co}_{3} \mathrm{O}_{4}\right.$ /graphene). The morphological characterizations revealed uniform, seamless coverage of a layer over the tip and nanoporous flower-like $\mathrm{Co}_{3} \mathrm{O}_{4}$ nanostructures (Fig. 8a-c). Using bare graphene as a counter electrode and $\mathrm{Co}_{3} \mathrm{O}_{4}$ /graphene needles as a working electrode, the Co(rv) active species generated in alkaline medium electrochemically detected the analyte glucose with a detection limit of $<10 \mu \mathrm{M}$ and a linear range of 50 to $300 \mathrm{mM}$ at $0.6 \mathrm{~V}$ vs. $\mathrm{Ag} / \mathrm{AgCl}$ under alkaline conditions. The 3D local detection with the high spatial resolution of the fabricated micropipette tip electrode may potentially replace conventional electrodes. ${ }^{\mathbf{1 1 0}}$

$\mathrm{Li}$ et al. acquired a $\mathrm{Co}_{3} \mathrm{O}_{4} /$ ErGO composite through an electrodeposition technique with varying deposition times of $\mathrm{Co}_{3} \mathrm{O}_{4}$ nanoparticles. The as-prepared hybrid exhibited evenly distributed $\mathrm{Co}_{3} \mathrm{O}_{4}$ nanoparticles over curled graphene sheets with an average size of $<100 \mathrm{~nm}$. Electrochemical impedance studies revealed that $\mathrm{Co}_{3} \mathrm{O}_{4} / \mathrm{ErGO} / \mathrm{GCE}$ exhibited a charge transfer resistance of $99 \Omega$, which is lower than that of the individual components $\mathrm{Co}_{3} \mathrm{O}_{4}$ and ErGO, owing to the efficient electrical network of the prepared composite. The electrochemical oxidation of glucose was achieved in alkaline conditions at $\mathrm{Co}_{3} \mathrm{O}_{4} / \mathrm{ErGO} / \mathrm{GCE}$, and an increase in $\mathrm{pH}$ negatively shifted the redox couple of $\mathrm{Co}_{3} \mathrm{O}_{4}(0.05 \mathrm{M} \mathrm{NaOH})$, specifying the requirement of a lower applied potential of $0.6 \mathrm{~V} v s$. SCE for amperometric sensing. This composite provided rapid glucose detection in human urine samples, with a recovery of $95.5 \%$ to $102.0 \% .{ }^{111}$ Other significant efforts devoted to NEGS containing $\mathrm{Co}_{3} \mathrm{O}_{4}$ /graphene/GCE are described in Table 2. ${ }^{112}$

NEGS was constructed with a nickel oxide (NiO) hollow spheres/rGO composite via a simple chemical reduction technique, in which the NiO hollow spheres were prepared using ethanol and urea as structure-directing agents. NiO hollow spheres were homogenously dispersed on graphene with a shell thickness of $2 \mu \mathrm{m}$ and a large void of $2 \mu \mathrm{m}$. The electrocatalytic glucose oxidation studies at $\mathrm{NiO} / \mathrm{rGO} / \mathrm{GCE}$ were achieved by $\mathrm{CV}$, in which glucose was oxidized into gluconolactone and gluconic acid in alkaline medium via the $\mathrm{Ni}$ (III) electroactive species. $\mathrm{NiO} /$ $\mathrm{rGO} / \mathrm{GCE}$ exhibited a high sensitivity of $3796 \mu \mathrm{A} \mathrm{mM} \mathrm{m}^{-1} \mathrm{~cm}^{-2}$, which is higher than that of NiOH/GCE $\left(2721 \mu \mathrm{A} \mathrm{mM}^{-1} \mathrm{~cm}^{-2}\right)$. The high electrocatalytic activity of the resulting composite is due to the porous nature of the NiO hollow spheres on rGO, which provided a 2D network for electronic transition and a large surface area. The material showed better results in human blood serum analysis, with an RSD of $2.76 \%$ on glucose spotting. ${ }^{113}$ The fabrication of NEGS using NiO/graphene dispersed with deoxyribonucleic acid (DNA) was achieved by an ultra-sonication-assisted self-assembly approach. The morphological studies depicted that the NiO/DNA/graphene hybrid exhibited highly dense NiO nanoparticles in which DNA served as a surfactant amphiphilic polymer molecule, which enhanced the dispersion of NiO nanoparticles on the surface of graphene. Traditionally, graphene-combined materials are difficult to disperse in aqueous solution due to the absence of functional groups. Interestingly, incorporation of DNA in the graphene matrix via GNS-sugar-phosphate skeleton interactions increased the stability of the as-prepared NiO/DNA/ graphene material, with better dispersion in aqueous medium. The active $\mathrm{NiOOH}$ species generated under alkaline conditions at NiO/DNA/graphene enhanced the peak current density after the addition of glucose, which was attributed to the electrooxidation of glucose to gluconolactone. NiO/DNA/graphene/ GCE exhibited good amperometric response under alkaline conditions at $0.6 \mathrm{~V}$ vs. $\mathrm{Ag} / \mathrm{AgCl}$, which was achieved by the enhanced electron transfer channels of DNA and graphene. ${ }^{\mathbf{1 4}}$

Sun et al. developed Qu (flavonoids)-stabilized Ni(II) nanoparticles (Ni(II)-Qu)-anchored graphene as an electrochemical probe for NEGS, in which Qu served as a complexant and enabled the facile and stable attachment of Ni(II) ions. The $\mathrm{Ni}(\mathrm{III})$ active species generated under alkaline conditions electrooxidized glucose and provided rapid amperometric response; the recovery obtained in the range of $94.7 \%$ to $103 \%$ in real samples demonstrated its practical applications. ${ }^{115}$ Belkhalfa et al. exploited electrophoretic deposition (EPD) for the preparation of thin films comprising insulin-impregnated nickel(II) hydroxide $\left(\mathrm{Ni}(\mathrm{OH})_{2}\right)$ nanostructures/rGO on an $\mathrm{Au}$ electrode. The higher release of insulin was attributed to the integration of insulin into the electrophoretically deposited thin film, resulting in impressive performance of the fabricated electrode. The possibility of the development of multifunctional coatings by EPD, along with the simple processes, uniformly deposited films and good control of the deposited film thickness, confirmed this approach to be a favorable advancement over 
conventional strategies. ${ }^{116}$ Zhang et al. developed a $\mathrm{NiO}$ nanofibers/rGO composite, and the morphological studies revealed that $\mathrm{NiO}$ nanofibers with a diameter of $350 \mathrm{~nm}$ were effectively anchored on $\mathrm{rGO}$ surface. $\mathrm{NiO} / \mathrm{rGO} / \mathrm{GCE}$ exhibited a response time, linear response, detection limit and sensitivity of $5 \mathrm{~s}, 2.0 \mu \mathrm{M}$ to $0.60 \mathrm{mM}, 0.77 \mu \mathrm{M}$ and $1100 \mu \mathrm{A} \mathrm{mM}{ }^{-1} \mathrm{~cm}^{-2}$, respectively, toward glucose detection under alkaline conditions at $0.6 \mathrm{~V} v s$. $\mathrm{Ag} / \mathrm{AgCl}$. The practicability of $\mathrm{NiO} / \mathrm{rGO} / \mathrm{GCE}$ was also validated from its good recovery rate of 5.0 to 5.4 in human blood serum. ${ }^{117}$ Subramanian et al. explored the arrangement of $\mathrm{Ni}(\mathrm{OH})_{2}$ nanostructures over rGO sheets using a one-pot electrophoretic deposition process. Characterization demonstrated the formation of porous $\mathrm{Ni}(\mathrm{OH})_{2} / \mathrm{rGO}$ films with optimized thicknesses with the aid of a DC potential of $+50 \mathrm{~V}$ for 10 to $60 \mathrm{~s}$. Under alkaline conditions, the $\mathrm{Ni}(\mathrm{OH})_{2} / \mathrm{rGO}$ film exhibited two crystalline phases, including hydrated $\alpha-\mathrm{Ni}(\mathrm{OH})_{2}$ and anhydrous $\beta-\mathrm{Ni}(\mathrm{OH})_{2}$ forms; after a couple of cycles, the oxidation peaks were dominated by the $\beta-\mathrm{Ni}(\mathrm{OH})_{2}$ form, and the glucose was electrooxidized by the active $\mathrm{Ni}(\mathrm{III})$ species. The amperometric results depicted that the $\mathrm{Ni}(\mathrm{OH})_{2} / \mathrm{rGO}$ film exhibited a response time, sensitivity, and detection limit of $<7 \mathrm{~s}, 11.4 \pm 0.3 \mathrm{~mA} \mathrm{mM} \mathrm{cm}^{-1}$ and $15 \mu \mathrm{M}$, respectively, at $0.6 \mathrm{~V}$ vs. $\mathrm{Ag} / \mathrm{AgCl}$. The good mechanical adherence of the matrix provided via electrophoretic deposition yielded durable electrode materials for NEGS and could be considered as an effective substitute for the drop-casting technique. ${ }^{118}$ The other significant efforts devoted to NEGS containing various $\mathrm{NiO} /$ graphene-based composites are provided in Table 2. ${ }^{119-123}$

Ye et al. acquired quantum-sized tin oxide $\left(\mathrm{SnO}_{2}\right)$ nanocrystals anchored on GO sheets for NEGS applications, and the mean diameter of the $\mathrm{SnO}_{2}$ nanocrystals anchored over GO sheets was found to be 2 to $5 \mathrm{~nm}$. The number of electrons, surface hydroxyl groups and defects of $\mathrm{SnO}_{x}$ effectively attacked the oxic carbon atoms of GO sheets. Specifically, $\mathrm{SnO}_{x}$ shared electrons with the generated $\mathrm{sp}^{2}$ hybridized carbon atoms and generated blue-shifts in the remaining $\mathrm{C}-\mathrm{OH}$ stretching peaks, which was also useful to maintain the chemical reactivity of the $\mathrm{SnO}_{x}$ crystals. The electrons shared from the $\mathrm{SnO}_{x}$ nanostructures effectively reduced GO to $\mathrm{rGO}$ and maintained good chemical reactivity in the crystallization process of $\mathrm{SnO}_{x} \cdot \mathrm{SnO}_{2} /$ rGO/GCE exhibited a sensitivity, linear range, and detection limit of $1.93 \mathrm{~A} \mathrm{M}^{-1} \mathrm{~cm}^{-2}, 0.05$ to $0.5 \mathrm{mM}$ and $13.35 \mu \mathrm{M}$, respectively, under alkaline conditions at $0.8 \mathrm{~V} v s . \mathrm{Ag} / \mathrm{AgCl}$, owing to the excellent electron transfer capability of the $\mathrm{SnO}_{2} /$ rGO composite. Moreover, $\mathrm{SnO}_{2} / \mathrm{rGO} / \mathrm{GCE}$ effectively detected glucose in human serum without any interfering effects from various bio-molecules. ${ }^{124}$

The significant electrochemical results obtained in nonprecious metal oxides/graphene-equipped NEGS are provided in Table $2 .^{71,90-124}$

\subsection{Alloy/bimetallic/hybrid metal nanostructures/graphene composites}

Owing to the synergistic effects of two metals, bimetallic systems could bring forth improvements in NEGS performance associated with a reduction in the interference and poisoning effects of electrodes and bimetallic systems, including alloys and metal/metal oxide composites. The combination of bimetallic systems with graphene has been widely practiced in NEGS, and the significant achievements in this area are discussed as follows:

A graphene-supported $\mathrm{Pt}-\mathrm{Ni}$ nanocomposite (Pt-Ni/ graphene) was prepared through an electrochemical technique; morphological studies depicted that spherical Pt-Ni (80 $\mathrm{nm}$ ) nanoparticles were uniformly anchored on the rGO sheets, where the rGO sheets enabled better dispersion of the bimetallic nanoparticles. The electrocatalytic activity of the fabricated $\mathrm{Pt}-\mathrm{Ni} / \mathrm{rGO} / \mathrm{GCE}$ was evaluated by $\mathrm{CV}$ under neutral conditions; it exhibited distinct peaks at -0.35 and $0.55 \mathrm{~V} v s$. $\mathrm{Ag} / \mathrm{AgCl}$, ascribed to the formation of glucose intermediates and the oxidation of intermediates to gluconic acid, respectively (Fig. 9a). Pt-Ni/ErGO/GCE exhibited a higher oxidation current density in comparison with $\mathrm{Pt}-\mathrm{Ni} / \mathrm{GCE}, \mathrm{Pt}-\mathrm{Ni} / \mathrm{CrGO} / \mathrm{GCE}$ (CrGO: chemically reduced GO) and $\mathrm{Pt}-\mathrm{Ni} / \mathrm{SWCNT/GCE}$ (Fig. 9a), owing to the specified spatial distribution of the PtNi nanoparticles, which was attributed to the continuous electrical network provided via the ErGO surface. The severe drawbacks of chemically reduced GO, such as an uneven patchy structure and low affinity toward metal nanoparticles, were effectively overcome by ErGO, which enabled the maximum electrocatalytic oxidation of glucose at the ErGO-based composite. $\mathrm{Pt}-\mathrm{Ni} / \mathrm{CrGO} / \mathrm{GCE}$ exhibited more negative potential and higher current densities in linear sweep voltammetry (LSV) toward glucose electrooxidation in comparison with the other studied electrodes (Fig. 9b). The chronoamperometric results revealed that the poisoning produced from the dehydrogenated intermediates at $\mathrm{Pt} / \mathrm{ErGO} / \mathrm{GCE}$ was overcome by $\mathrm{Pt}-\mathrm{Ni} / \mathrm{ErGO} /$ GCE, as substantiated from the long-term stability, owing to the "bifunctional effect" and "ligand or electronic effect" of the
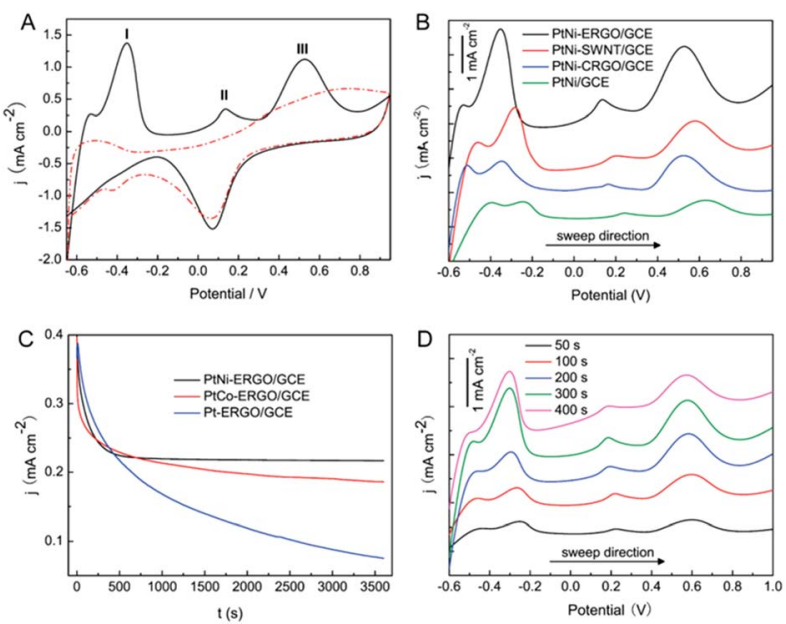

Fig. 9 (A) CV responses of Pt-Ni/ErGO/GCE under the absence (red dash line) and presence (black solid line) of $10 \mathrm{mM}$ glucose, (B) LSV responses of the studied GCEs in $10 \mathrm{mM}$ glucose at $10 \mathrm{mV} \mathrm{s}^{-1}$, (C) chronoamperometric curves of the studied GCEs at scan rate $0.35 \mathrm{~V}$ vs. $\mathrm{Ag} / \mathrm{AgCl}$, and (D) LSV responses of $\mathrm{Pt}-\mathrm{Ni} / \mathrm{ErGO} / \mathrm{GCE}$ at different deposition times of metal nanoparticles toward $10 \mathrm{mM}$ glucose at $10 \mathrm{mV} \mathrm{s}^{-1}$ scan rate (reproduced with permission from American Chemical Society; Gao et al., 2011). 
Pt-Ni nanostructures. It was concluded from the LSV curves (Fig. 9c) that the electrodeposition time of $300 \mathrm{~s}$ provided the maximum electrooxidation of glucose, owing to the optimal diameter of the Pt-Ni nanoparticles (Fig. 9d). The amperometric studies examined at $-0.35 \mathrm{~V}$ vs. $\mathrm{Ag} / \mathrm{AgCl}$ demonstrated its potential applications, and a RSD of less than $5 \%$ was obtained for practical analysis of human urine samples. The wide application of graphene in glucose sensors was proven by comparing the electrochemical performance of graphene with that of SWCNTs. Owing to the homogenous planar electronic structure of graphene, the metal nanoparticles were uniformly distributed over its surface, whereas uneven distribution and aggregation of metal nanoparticles were observed over the surface of SWCNTs due to the uniform electrical structures along its tips and side walls, demonstrating the importance of graphene in NEGS. ${ }^{125} \mathrm{Pt}-\mathrm{Au} / \mathrm{manganese}$ oxide $\left(\mathrm{Pt}-\mathrm{Au} / \mathrm{MnO}_{2}\right)$ anchored on flexible free-standing graphene paper (GP) (Pt-Au/ $\mathrm{MnO}_{2} / \mathrm{GP}$ ) was prepared using a template-free electrodeposition technique by exploiting a polytetrafluoroethylene (PTFE) casting mold. The morphological features of the prepared hybrid revealed wrinkled GP with a thickness of $10 \mu \mathrm{m}$ and a coral structure with a length of 30 to $100 \mathrm{~nm}$ which consisted of interlinked nanorods with a mean diameter of $20 \mathrm{~nm}$. The electrocatalytic behaviour of the prepared hybrid toward glucose oxidation was analyzed using CV in neutral medium, and $\mathrm{Pt}-\mathrm{Au} / \mathrm{MnO}_{2} / \mathrm{GP}$ revealed multiple oxidation peaks at $0,0.3$ and $-0.2 \mathrm{~V}$ vs. $\mathrm{Ag} / \mathrm{AgCl}$, depicting the formation of enediol intermediates due to the electrosorption of glucose on the catalyst surface, the electrooxidation of those intermediates to gluconolactone or gluconic acid and the reduction of the metal
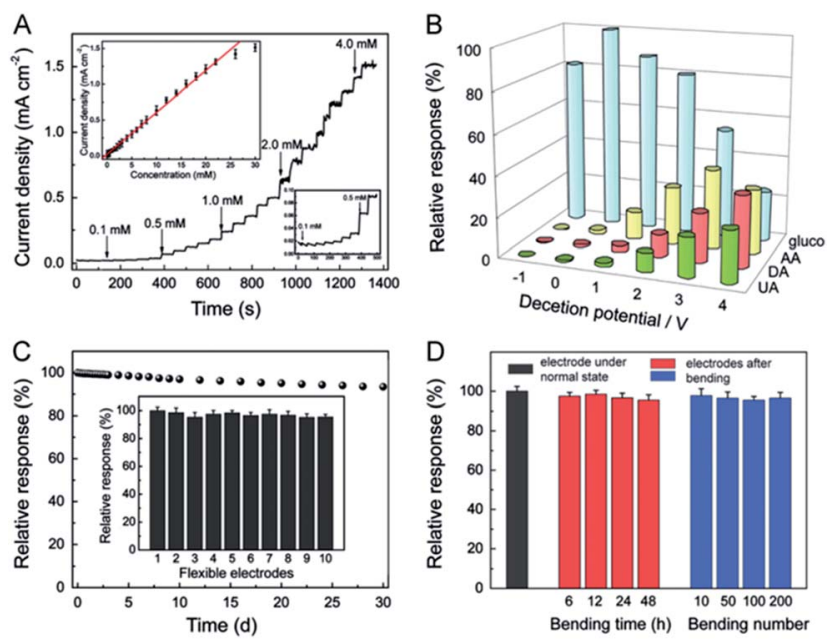

Fig. 10 (A) Amperometric responses of $\mathrm{Pt}-\mathrm{Au} / \mathrm{MnO}_{2} / \mathrm{GP}$ with the consecutive addition of glucose at different concentrations in PBS (inset: correlating calibration curves), (B) amperometric responses of $\mathrm{Pt}-\mathrm{Au} / \mathrm{MnO}_{2} / \mathrm{GP}$ toward $5 \mathrm{mM}$ glucose and $0.1 \mathrm{mM} \cup \mathrm{A}$, DA and $\mathrm{AA}$ interference as a function of applied potential, (C) current responses of $\mathrm{Pt}-\mathrm{Au} / \mathrm{MnO}_{2} / \mathrm{GP}$ toward $5 \mathrm{mM}$ glucose as a function of time (inset: current responses of ten different $\mathrm{Pt}-\mathrm{Au} / \mathrm{MnO}_{2} / \mathrm{GP}$ samples), and (D) influence of bending time and bending number on the current responses of $\mathrm{Pt}-\mathrm{Au} / \mathrm{MnO}_{2} / \mathrm{GP}$ toward $5 \mathrm{mM}$ glucose (reproduced with permission from Elsevier; Xiao et al. 2013). oxide, respectively. The excellent amperometric responses obtained at $\mathrm{Pt}-\mathrm{Au} / \mathrm{MnO}_{2} / \mathrm{GP}$ (Fig. 10a) toward glucose detection are attributed to the enhancement of electron transfer achieved via the synergistic combination of its various components. Owing to the relatively negative applied potential of $0 \mathrm{~V} v \mathrm{~s}$. Ag/ $\mathrm{AgCl}$, the amperometric responses of interference molecules, including AA, dopamine (DA) and UA, were effectively avoided at $\mathrm{Pt}-\mathrm{Au} / \mathrm{MnO}_{2} / \mathrm{GP}$ (Fig. 10b). The fabricated sensor maintained $95 \%$ of its initial sensitivity after 30 days of operation, which validated its stability (Fig. 10c). The flexibility of Pt-Au/ $\mathrm{MnO}_{2} /$ GP was determined by subjecting it to inward bending at $180^{\circ}$; the electrocatalytic activity of the prepared hybrid was the same as that of a non-bended electrode, which indicated the excellent mechanical stability of the fabricated electrode (Fig. 10d). The explicit advantages of GP, such as greater flexibility, mechanical robustness, uniform structure, and enhanced electrical conductivity and stability, associated with the superior electrocatalytic activity of the metal composite endowed with rapid performance in glucose sensors. ${ }^{\mathbf{1 2 6}}$

$\mathrm{Ni}-\mathrm{Co}$ nanoparticles decorated on $\mathrm{rGO}(\mathrm{Ni}-\mathrm{Co} / \mathrm{rGO})$ were prepared by exploiting an electrochemical technique, and the morphological studies of the as-prepared composite depicted that these nanocomposites contained well-patterned, $300 \mathrm{~nm}$ sized flower-like Ni-Co nanospheres on the rGO sheets. During the investigation of the electrocatalytic behaviour of these nanostructures using CV under alkaline conditions, oxidation peaks appeared at 0.27 and $0.50 \mathrm{~V} v s$. SCE; these were attributed to the conversion of glucose to gluconolactone with the reactive species of $\mathrm{NiOOH}$ and $\mathrm{CoO}_{2}$, respectively. The influence of the applied potential on glucose electrooxidation was measured with the aid of different applied potentials ranging from 0.3 to $0.6 \mathrm{~V} v s$. SCE, in which $0.5 \mathrm{~V} v s$. SCE was chosen as the optimized applied potential owing to its high and
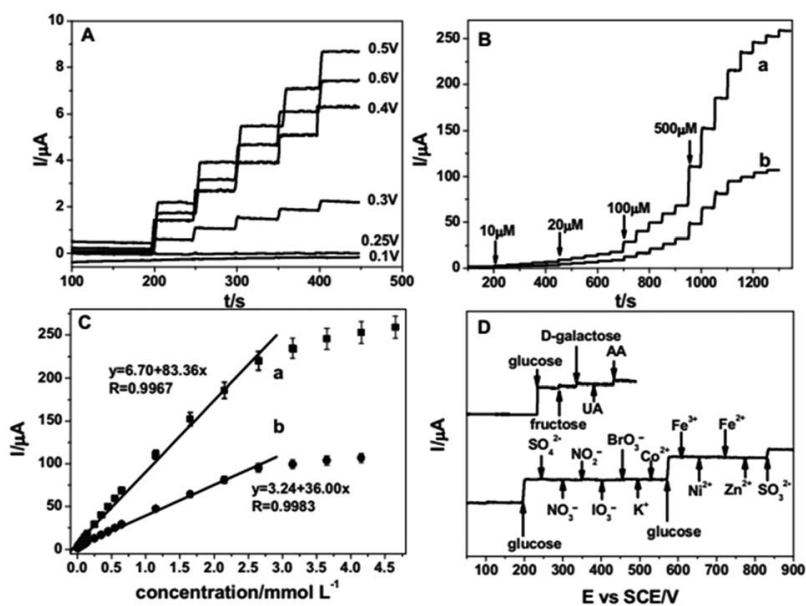

Fig. 11 (A) Amperometric responses of $\mathrm{Ni}-\mathrm{Co} / \mathrm{rGO} / \mathrm{GCE}$ toward $10 \mathrm{mM}$ glucose with different applied potentials, (B) amperometric responses of (a) $\mathrm{Ni}-\mathrm{Co} / \mathrm{rGO} / \mathrm{GCE}$ and (b) $\mathrm{Ni}-\mathrm{Co} / \mathrm{GCE}$ to the consecutive addition of glucose in $\mathrm{NaOH}$ at $0.5 \mathrm{~V}$ vs. SCE, (C) calibration plots of glucose concentration vs. steady-state current, and (D) interference study of $\mathrm{Ni}-\mathrm{Co} / \mathrm{rGO} / \mathrm{GCE}$ at $0.5 \mathrm{~V}$ vs. SCE (reproduced with permission from Elsevier; Wang et al. 2013). 
steady $I_{\mathrm{pa}}$ values (Fig. 11a). The influence of rGO on the electrocatalytic oxidation of glucose was clearly evidenced by the higher $I_{\mathrm{pa}}$ of $\mathrm{Ni}-\mathrm{Co} / \mathrm{rGO} / \mathrm{GCE}$ in comparison with $\mathrm{Ni}-\mathrm{Co} / \mathrm{GCE}$; this is due to the large surface area of rGO, which decreased the size of the Ni-Co nanoparticles. Moreover, the conjugation of Co enhanced the Ni by enabling it to reach a higher oxidation level and promoted higher oxidation with less $\beta$-phase $\mathrm{Ni}(\mathrm{OH})_{2}$ formation upon oxidation of glucose, which resulted in good sensitivity toward the glucose detection due to the synergetic effect between rGO and the Ni-Co nanospheres (Fig. 11b and c). The constructed $\mathrm{Ni}-\mathrm{Co} / \mathrm{rGO} / \mathrm{GCE}$ exhibited excellent selectivity toward glucose against a number of interference molecules (Fig. 11d), and its reliability was verified by real sample analysis of glucose in blood serum, with a RSD of $5.6 \% .^{127}$

A low cost screen-printed electrode (SPE) was modified with $\mathrm{Pt}-\mathrm{CuO}$ decorated rGO nanosheets for NEGS applications. Monoclinic $\mathrm{CuO}$ nanoflowers with $d$-spacing of $0.27 \mathrm{~nm}$ along with cubic crystalline Pt nanocubes with $d$-spacing of $0.12 \mathrm{~nm}$ were uniformly dispersed over the wrinkled GR sheets. The electrocatalytic behaviors of these nanostructures were investigated using CV; a glucose oxidation peak was exhibited at $0.35 \mathrm{~V}$ vs. $\mathrm{Ag} / \mathrm{AgCl}$, where $\mathrm{CuO}$ electrocatalyzed the oxidation of glucose in basic medium and Pt enhanced the sensitivity of the catalyst by its co-catalyst role, which increased the rate of electron transfer during glucose oxidation. Furthermore, $\mathrm{Pt}-\mathrm{CuO} / \mathrm{rGO} /$ SPE exhibited an enhanced oxidation peak current of $267 \mu \mathrm{A}$, which is greater than that of $\mathrm{CuO} / \mathrm{rGO} / \mathrm{SPE}$; this is due to the enhanced catalytic activity by the incorporation of the bimetallic species in the rGO matrix, where surface-confined kinetics toward glucose oxidation was observed. Also, the enhanced electron transfer observed at the fabricated electrode exhibited outstanding amperometric performance toward the electrochemical detection of glucose. ${ }^{128}$

A ternary composite comprising NiO nanoparticles with $\mathrm{Pt}$ nanoparticles was electrodeposited on ErGO and utilized as an electrochemical sensor probe for a NEGS. From morphological studies, it is clear that these interconnected NiO/Pt nanoparticles were homogenously dispersed on the surface of multilayer structured ErGO, which underwent irreversible agglomeration. The electrocatalytic behavior of $\mathrm{NiO} / \mathrm{Pt} / \mathrm{ErGO} /$ GCE was analyzed using CV in basic medium; the oxidation of glucose to gluconolactone was due to the electrocatalytic process driven by the NiOOH species, and the minimal amount of Pt catalyst present in the composite enhanced the catalytic effects of the NiO electrocatalyst during the course of glucose oxidation. The aforesaid composite demonstrated efficient amperometric sensing toward glucose at $0.6 \mathrm{~V} v s$. $\mathrm{Ag} / \mathrm{AgCl}$ due to the enhanced surface area, electron transfer and conductivity of $\mathrm{NiO} / \mathrm{Pt} / \mathrm{ErGO} / \mathrm{GCE}$. Furthermore, the high selectivity obtained at $\mathrm{NiO} / \mathrm{Pt} / \mathrm{ErGO} / \mathrm{GCE}$ was mainly due to the constructive characteristics of the isoelectronic point of NiO (10-11), which effectively repelled the negatively charged UA and AA (negative charge arises with the loss of protons in alkaline conditions) and ensured its selectivity. ${ }^{\mathbf{1 2 9}}$ A distinct 3D GR hydrogel (GRH) encapsulating $\mathrm{Pd}-\mathrm{Cu}$ nanoparticles was prepared by a hydrothermal technique, and the morphological characterizations depicted that the $\mathrm{Pd}-\mathrm{Cu}$ nanoparticles were homogenously distributed over the interlinked and porous 3D framework of the GR matrix. Under an applied potential of more than $0.30 \mathrm{~V}$ vs. SCE, the $\mathrm{Pd}-\mathrm{OH}_{\mathrm{ads}}$ species were generated at $\mathrm{Pd}_{66} \mathrm{Cu}_{34} / \mathrm{GRH}$ in alkaline solution (Fig. 12a), which facilitated the oxidation of glucose to gluconic acid or gluconolactone; the Pd-based catalysts were highly active compared to $\mathrm{Cu}$-based catalysts under alkaline conditions. On varying the proportion of $\mathrm{Pd}$ and $\mathrm{Cu}$, a higher molar ratio of $\mathrm{Pd}$ resulted in better electrocatalytic activity, where Pd served as a Lewis acid and the analyte glucose served as a Lewis base; subsequently, the feasible adsorption via non bonding electrons improved the electrocatalytic activity toward glucose oxidation. The dominant role of Pd compared to $\mathrm{Cu}$ in the $\mathrm{PdCu} / \mathrm{GRH}$ bimetallic hybrid was confirmed from the excellent electrochemical glucose oxidative properties of $\mathrm{Pd}_{66} \mathrm{Cu}_{34} / \mathrm{GRH}$ (Fig. 12b), which is purely attributed to the high reactivity of $\mathrm{Pd}$ compared to that of $\mathrm{Cu}$ under alkaline conditions. Under neutral conditions, the glucose oxidation signals were weakened at $\mathrm{Pd}-\mathrm{Cu} / \mathrm{GRH}$, which necessitated an alkaline environment for effectual electrooxidation (Fig. 12c). Upon chloride poisoning, Pd-Cu/GRH exhibited only a $5 \%$ to $10 \%$ reduction in oxidation current, demonstrating the robust stability of the prepared composite against the $\mathrm{Cl}^{-}$environment (Fig. 12d). The amperometric responses obtained at $-0.4 \mathrm{~V} v s$. SCE were found to be superior, and the porous 3D GRH framework with efficient oxygen functionalities promoted the nucleation of metal nanoparticles, the accessibility of glucose to the active sites and the dynamics of the reaction; this was highly beneficial for the electrocatalytic activity of the composite toward glucose detection. ${ }^{\mathbf{1 3 0}}$
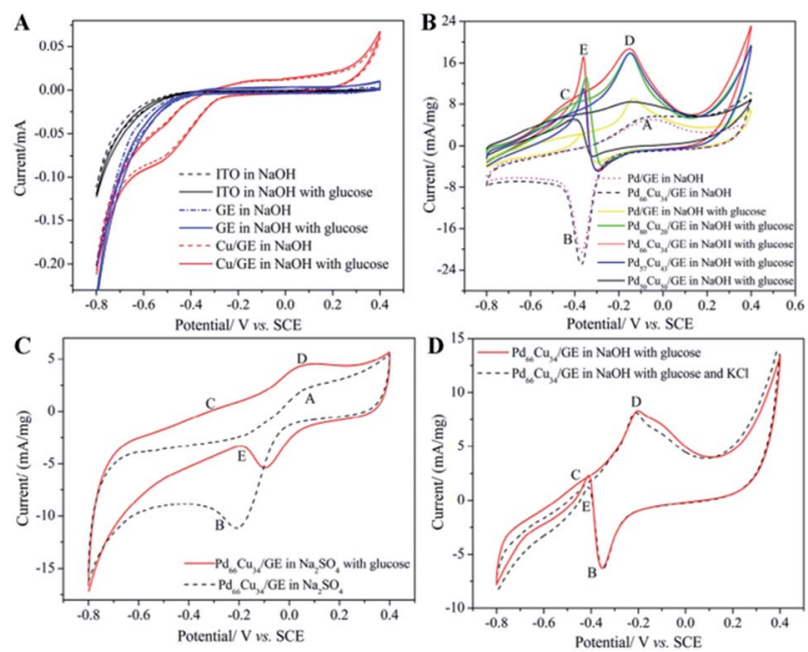

Fig. 12 (A) CV responses of studied GCEs under the absence and presence of $10 \mathrm{mM}$ glucose in $0.1 \mathrm{M} \mathrm{NaOH}$ with a scan rate of $50 \mathrm{mV}$ $\mathrm{s}^{-1}$, (B) CV responses of $\mathrm{Pd} / \mathrm{GE}$ and $\mathrm{Pd}_{x} \mathrm{Cu}_{y} / \mathrm{GE}$ series in the absence and presence of $10 \mathrm{mM}$ glucose in $0.1 \mathrm{M} \mathrm{NaOH}$ with a scan rate of $50 \mathrm{mV} \mathrm{s}^{-1}$, (C) CV responses of $\mathrm{Pd}_{66} \mathrm{Cu}_{34} / \mathrm{GE} / \mathrm{ITO}$ in the absence and presence of $10 \mathrm{mM}$ glucose in $0.1 \mathrm{M} \mathrm{Na}_{2} \mathrm{SO}_{4}$ with $50 \mathrm{mV} \mathrm{s}^{-1}$ scan rate and (D) CV responses of $\mathrm{Pd}_{66} \mathrm{Cu}_{34} / \mathrm{GE} / \mathrm{ITO}$ in $\mathrm{N}_{2}$-saturated $0.1 \mathrm{M}$ $\mathrm{NaOH}$ and $5 \mathrm{mM}$ glucose solution with and without $0.15 \mathrm{M} \mathrm{KCl}$ at 50 $\mathrm{mV} \mathrm{s}^{-1}$ scan rate (reproduced with permission from Elsevier; Yuan et al. 2014). 
By exploiting the galvanic replacement approach, novel hollow Pt-Ni nanostructure-anchored graphene (Pt-Ni/ graphene) was prepared at an ambient temperature; the replacement reaction between $\mathrm{PtCl}_{6}{ }^{2+}$ and $\mathrm{Ni}$ nanosheets generated $30 \mathrm{~nm}$-sized hollow Pt-Ni nanostructures. The electrochemical behavior of the fabricated Pt-Ni/GR/GCE was probed under neutral conditions; the authentic peak observed at $0.4 \mathrm{~V}$ vs. SCE confirmed the oxidation of the adsorbed intermediates to gluconolacotone or gluconic acid during the analysis of glucose. The hollow structured Pt-Ni/graphene/GCE exhibited a 3 fold-enhanced electrocatalytic current compared to the solid structured Pt-Ni/graphene, which depicted the unique performance of the hollow structures with greater accessibility of the analyte to its active sites along with its porous surface. The obtained superior amperometric performance is due to the presence of hollow formed $\mathrm{Pt}-\mathrm{Ni}$, with a large specific surface area, enhanced number of active sites, and intriguing catalytic properties; graphene played a vital role by increasing the density and distribution of the catalyst and enhanced the electrocatalytic activity via providing a continuous electrical network. Furthermore, the limitations of bare Pt nanoparticles, such as chloride poisoning, low stability and low sensitivity, were effectively overcome by the conjugation of $\mathrm{Ni}^{\mathbf{1}}{ }^{\mathbf{3 1}}$

Pt-Pd nanoparticles anchored on rGO sheets were synthesized using a microwave method with ionic liquid (IL, [VEIM] $\mathrm{BF}_{4}$ )-assisted reactions, where the addition of the IL improved the dispersion of Pt-Pd nanoparticles on the rGO sheets. Morphological characterizations of the $\mathrm{Pt}-\mathrm{Pd} / \mathrm{IL} / \mathrm{rGO}$ composite revealed that the Pt-Pd nanoparticles exhibited an average particle size of $3.4 \mathrm{~nm}$ with uniform distribution; meanwhile, the Pt-Pd nanoparticles exhibited an average diameter of $6.3 \mathrm{~nm}$ without the aid of IL, demonstrating the effectual role of $\mathrm{IL}$, in which the electrostatic interaction exerted between IL ions and $\left(\mathrm{PtCl}_{6}\right)^{2-}$ and $\left(\mathrm{PdCl}_{4}\right)^{2-}$ species governed of positive charges the uniform dispersion of Pt-Pd nanoparticles over of negative charges the surface of the rGO sheets. The electrochemical analysis achieved via CV revealed that the sequential steps, including the electroadsorption of glucose, glucose oxidation, intermediate formation and further oxidation of the intermediate, for the generation of gluconolactone occurred at -0.35 , 0.15 and $0.3 \mathrm{~V}$ vs. $\mathrm{Ag} / \mathrm{AgCl}$, respectively. $\mathrm{Pt}(50)-\mathrm{Pd}(50) / \mathrm{IL}(100) /$ $\mathrm{rGO} / \mathrm{GCE}$, with a surface area of $6.61 \mathrm{~cm}^{2}$, afforded enhanced peak current density over $\mathrm{Pt}(50)-\mathrm{Pd}(50) / \mathrm{rGO} / \mathrm{GCE}$ with a surface area of $3.98 \mathrm{~cm}^{2}$, clearly evincing the role of IL in governing the uniform distribution of metal nanoparticles and the catalytic activity. A high resistance to $\mathrm{Cl}^{-}$ions was accessed for $\mathrm{Pt}(50)-$ $\mathrm{Pd}(50) / \mathrm{IL}(100) / \mathrm{rGO} / \mathrm{GCE}$ in $50 \mathrm{mM}$ glucose in the presence and absence of $0.15 \mathrm{M} \mathrm{NaCl}$ (Fig. 13a). The optimized in 0.10 M PBS potential for the effectual glucose electrooxidation was found to be $0 \mathrm{~V} v s$. $\mathrm{Ag} / \mathrm{AgCl}$ (Fig. 13b), and the influences of interferences molecules, including UA, DA and AA, were minimized at $0 \mathrm{~V} v s$. $\mathrm{Ag} / \mathrm{AgCl}$ in comparison with the other applied potentials (Fig. 13c and d). Pt(50)-Pd(50)/IL(100)/rGO/GCE demonstrated a rapid amperometric responses at $0 \mathrm{~V} v s$. $\mathrm{Ag} / \mathrm{AgCl}$ under neutral conditions. ${ }^{132}$ Pt-Pd nanocubes-anchored graphene was prepared using $\mathrm{N}, \mathrm{N}$-dimethylformamide as a reducing agent and surface confining agent by a surfactant free formation technique; morphological characterization revealed that $8 \mathrm{~nm}$ sized Pt-Pd nanocubes in an atomic ratio of $1: 1$ were decorated on the graphene, where $\mathrm{Pt}-\mathrm{Pd} /$ graphene $\left(0.34 \mathrm{~cm}^{2}\right)$ has a greater electroactive surface area than Pd/graphene $\left(0.15 \mathrm{~cm}^{2}\right)$ and $\mathrm{Pt} /$ graphene $\left(0.28 \mathrm{~cm}^{2}\right)$. The electrocatalytic oxidation of glucose was occurred at $-0.28 \mathrm{~V}$ vs. SCE for the fabricated Pt$\mathrm{Pd} /$ graphene/GCE, which is attributed to the electrosorption of glucose and the generation of intermediates; the intermediates were oxidized to gluconic acid at $0.2 \mathrm{~V}$ vs. SCE under neutral conditions. Amperometric analysis studies revealed that the alloy particles prepared from a 1:1 molar ratio of $\mathrm{Pt}$ and $\mathrm{Pd}$ revealed the enhanced electrochemical detection in comparison with $1: 5,1: 3,3: 1$ and $5: 1$ molar ratios of $\mathrm{Pt}$ and $\mathrm{Pd}$; this is due to the presence of sufficient Pd for the oxidative removal of intermediates electroadsorbed on the active sites of Pt, which enhanced the sensitivity. The synergetic effects exerted between $\mathrm{Pt}$ and Pd facilitated the creation of vacancies by Pd in the Pt dband, yielding enhanced electrocatalytic activity, whereas graphene enhanced the conductivity and distribution of the prepared metal nanoparticles. ${ }^{133} \mathrm{Pd}-\mathrm{NiO}$ with nile-blue (NB) functionalized rGO was achieved by a simple chemical reduction technique, and $\mathrm{Pd}$ nanoparticles were incorporated on $\mathrm{NiO} / \mathrm{NB} / \mathrm{rGO}$ via a galvanic replacement method. NB served as a redox mediator in the electrocatalytic process and prevented the restacking of rGO sheets. Morphological characterizations revealed that the $\mathrm{NiO}$ nanoparticles were effectively piled over the surface of the NB/rGO sheets, and the Pd sites were partially reduced with the active $\mathrm{Ni}$ sites. $\mathrm{CV}$ studies revealed that the glucose oxidation occurs at $-0.04 \mathrm{~V}$ vs. $\mathrm{Ag} / \mathrm{AgCl}$ by means of

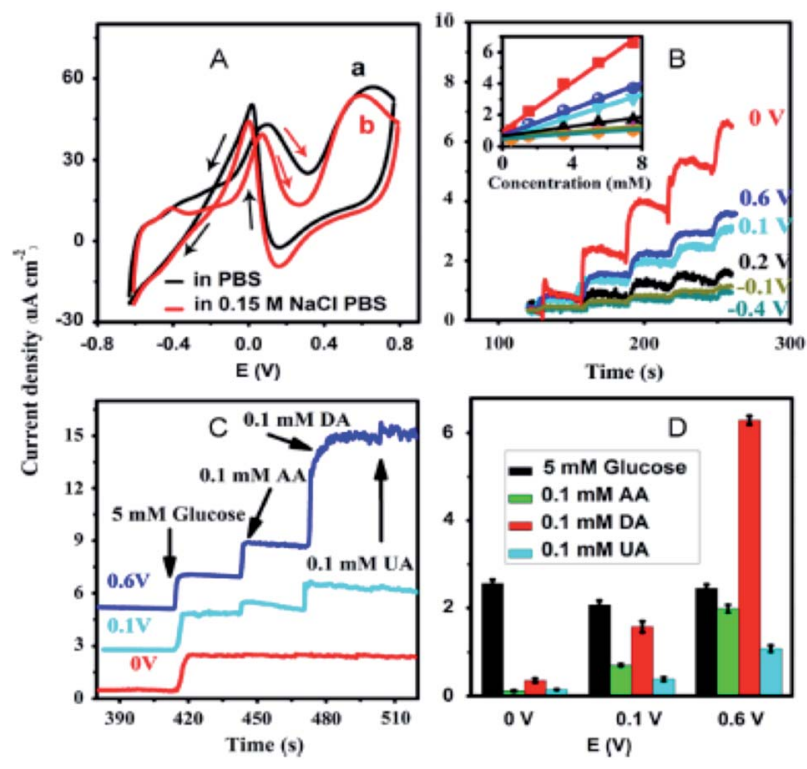

Fig. 13 (A) CV responses of $\mathrm{Pt}(50)-\mathrm{Pd}(50) / \mathrm{IL}(100) / \mathrm{rGO} / \mathrm{GCE}$ in $0.1 \mathrm{M}$ PBS in the presence and absence of $0.15 \mathrm{M} \mathrm{NaCl}$ at $5 \mathrm{mV} \mathrm{s}^{-1}$, (B) CV responses of $\mathrm{Pt}(50)-\mathrm{Pd}(50) / \mathrm{IL}(100) / \mathrm{rGO} / \mathrm{GCE}$ to the consecutive addition of glucose at different potentials (inset: corresponding calibration plots) and (C and D) amperometric responses of $\mathrm{Pt}(50)$ $\mathrm{Pd}(50) / \mathrm{IL}(100) / \mathrm{rGO} / \mathrm{GCE}$ toward glucose and other interference molecules at different applied potentials (reproduced with permission from Elsevier; Li et al., 2014). 
electroactive $\mathrm{Pd}(\mathrm{OH})_{X}$ and $\mathrm{Ni}(\mathrm{OH})_{X}$ species under alkaline conditions; $\mathrm{Pd} / \mathrm{NiO} / \mathrm{NB} / \mathrm{rGO} / \mathrm{CPE}$ showed higher electrocatalytic activity than $\mathrm{Pd} / \mathrm{NiO} / \mathrm{rGO} / \mathrm{CPE}$ owing to the superior electron transport role of NB and the homogeneous distribution of metal nanoparticles, which served as a redox indicator. The composite exhibited a broad linear range and lower detection limit toward glucose oxidation. ${ }^{\mathbf{1 3 4}}$ A disposable SPE comprising $\mathrm{Au}-\mathrm{CuO} / \mathrm{rGO}$ was prepared by a single-step chemical process; morphological characterization revealed that the combined $\mathrm{Au}$ nanoparticles and $\mathrm{CuO}$ nanorod bundles were evenly decorated on the wrinkled surface of the rGO sheets. The voltammetric analysis in basic medium revealed that the glucose oxidation was occurred at $0.4 \mathrm{~V}$ vs. $\mathrm{Ag} / \mathrm{AgCl}$, where $\mathrm{Cu}(\mathrm{III})$ electrooxidized glucose to gluconolactone. Moreover, the $I_{\mathrm{pa}}$ obtained at $\mathrm{Au}-\mathrm{CuO} / \mathrm{rGO} / \mathrm{SPE}$ was $312 \mu \mathrm{A}$, amidst a greater sensitivity of $2356 \mu \mathrm{A} \mathrm{mM}^{-1} \mathrm{~cm}^{-2}$ over $\mathrm{CuO} / \mathrm{rGO} / \mathrm{SPE}$; this indicates the efficiency of the bimetallic nanocomposite. The optimum working potential was probed via amperometric analysis at $+0.60 \mathrm{~V}$ vs. $\mathrm{Ag} / \mathrm{AgCl}$, where the composite showed a linear range of $1 \mu \mathrm{M}$ to $12 \mathrm{mM}$ and a limit of detection of $0.1 \mu \mathrm{M}$ owing to the combined effects of the synthesized material, in which $\mathrm{Cu}$ (III) catalyzed the oxidation of glucose with rGO and provided the advantages of large surface area and improved electrical conductivity. This fabricated material provided improved performance toward real blood glucose sample analysis, i.e., analyzing glucose human blood serum, with reliable glucose detection. ${ }^{\mathbf{1 3 5}}$ Primitive hexagonal Pd nanoparticles $(10 \mathrm{~nm})$ and elongated monoclinic $\mathrm{CuO}$ nanoparticles $(\sim 80 \mathrm{~nm})$ homogeneously anchored on rGO sheets $(\mathrm{Pd}-\mathrm{CuO} / \mathrm{rGO})$ were prepared using a one-step chemical reduction technique. The electrochemical properties of Pd$\mathrm{CuO} / \mathrm{rGO} / \mathrm{SPE}$ were evaluated using LSV in basic medium; it was found that the oxidation of glucose was achieved by the active $\mathrm{CuOOH}$ species, and the $E_{\mathrm{pa}}$ was observed at $0.35 \mathrm{~V} v s$. $\mathrm{Ag} / \mathrm{AgCl}$. The synergistic effects of $\mathrm{Pd}$ and $\mathrm{CuO}$ with greater capacitance and lower resistance facilitated excellent amperometric behavior toward glucose detection. The real sample analysis for blood serum at Pd-CuO/rGO/SPE showed a RSD of less than $3.2 \% .{ }^{136} \mathrm{NiCoS}_{2}$ nanoparticles anchored on porous rGO (PrGO) sheets were prepared using a hydrothermal technique and utilized as an electrocatalyst for NEGS applications. $\mathrm{NiCoS}_{2} /$ PrGO composites were also prepared using a hydrothermal technique; various molar ratios of $\mathrm{Ni}$ and Co were used, wherein the $\mathrm{Ni}_{0.31} \mathrm{Co}_{0.69} \mathrm{~S}_{2}$ proportioned material showed excellent electrocatalytic activity toward glucose oxidation due to its low charge transfer resistance. Morphological studies revealed that the $\mathrm{Ni}_{0.31} \mathrm{Co}_{0.69} \mathrm{~S}_{2}$ nanoparticles were uniformly distributed on the micrometer-sized porous interconnected framework of PrGO; the high diffusion coefficient and greater catalytic rate constant on the porous structure of rGO increased the rate of transmission of ions and electrons during the electrochemical glucose oxidation reaction sequence. CV characterization revealed that the oxidation of glucose in basic medium was observed at 0.35 and $0.22 \mathrm{~V}$ vs. $\mathrm{Ag} / \mathrm{AgCl}$, representing the redox reaction of $\mathrm{Ni}_{0.31} \mathrm{Co}_{0.69} \mathrm{~S}_{2} / \mathrm{Ni}_{0.31} \mathrm{Co}_{0.69} \mathrm{~S}_{2} \mathrm{OH}$ and the inclusion of glucose; the higher anodic peak current represented the oxidation of glucose to gluconolactone by the reactive catalytic species $\mathrm{Ni}_{0.31} \mathrm{Co}_{0.69} \mathrm{~S}_{2} \mathrm{OH}$. The doping of $\mathrm{Co}$ enhanced the conductivity, and the interlinked porous graphene matrix reduced the diffusion distance between glucose and the active surface of the $\mathrm{Ni}_{0.31} \mathrm{Co}_{0.69} \mathrm{~S}_{2}$ nanoparticles. The highly conducting nature of PrGO offered rapid transmittance of electrons from the reaction sites to the electrode surface, which promoted the glucose oxidation kinetics. ${ }^{\mathbf{1 3 7}}$ Alloy/bi-metal/hybrid metal nanoparticles/graphene-equipped NEGS with significant electrochemical performance are provided in Table $3 .{ }^{127-139}$ By the combination of two excellent modalities as metal/metal oxide nanostructures/graphene composites, excellent electrical, electrochemical, and optical properties were achieved which were superior to those of the individual components. The good contact exerted between graphene and the metal nanostructures effectively prevented irreversible agglomeration and volume changes and restacking of metal nanoparticles and graphene, respectively, resulting in enhanced stability. The aforesaid composites yielded increased electrochemically available surface areas and electrical conductivities for beneficial analyte adsorption and electron mobility, respectively, which collectively enhanced their sensitivity toward glucose oxidation. The electrically conductive $2 \mathrm{D} / 3 \mathrm{D}$ graphene template facilitated the electrical properties and charge transportation paths of the pure metal oxides. The unavoidable alteration observed in the native electronic structure and physical properties of graphene in covalent functionalization was effectively addressed by the non-covalent functionalization exerted between the metal nanostructures and graphene, which preserved the intrinsic properties of the original $\mathrm{sp}^{2}$ conjugated structure of graphene with considerable electrical conductivity. However, the non-covalent functionalization of metal nanostructures with graphene exhibited certain constraints, including low stability, leaching out of metallic active sites, chloride poisoning, and low hydrophilicity, which limited the durability of the NEGS. This could be overcome with an effectual covalent functionalization of metal nanoparticles on the graphene matrix via novel approaches.

In general, the interfacial properties between the electrode and the analyte of interest in metal/metal oxide nanoparticles/ graphene platform-based NEGS are purely governed by the dispersibility and interface interaction between the graphene and metallic nanoparticles. The oxygen-rich functionalities of graphene-based platforms are highly essential for good dispersion, which serves as an excellent template suspension for the anchoring of well-dispersed ultra-fine nanostructures with improved interfacial interactions and electrical contacts between metal/metal oxide nanostructures and graphene. Hence, immense research activities on the above topic must be performed to realize dispersion and interface contact between the metal/metal oxide nanostructures and graphene. Owing to the van der Waals interactions between the bi and trilayer graphene sheets, the unique electronic properties of graphene are deteriorated, which yields poor interface interactions between the metal nanoparticles and graphene. Hence, the utilization of single layer graphene in the preparation of metal/metal oxide nanoparticles/graphene composites is highly beneficial for improved interaction between the metal nanostructures and graphene compared to bi and trilayer graphene. The dispersion 


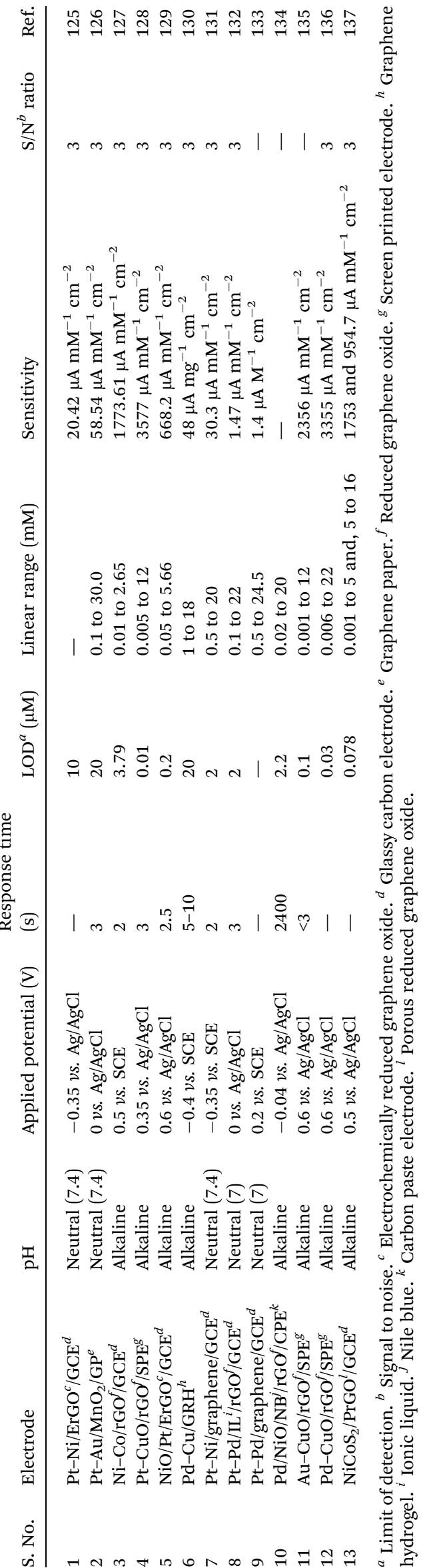

and interfacial contact between the metal nanoparticles and graphene could also be enhanced by covalent (organic molecules, chromophores and polymers) and non-covalent $\left(\pi-\pi, \mathrm{H}_{-}\right.$ $\pi$, cation/anion- $\pi$ and van der Waals) functionalizations of graphene sheets followed by the nucleation and growth of metal nanostructures.

\subsection{Polymer/graphene composites}

Polymer/graphene based platform composites are intriguing NEGS probes owing to their excellent mechanical, electrical and thermal properties, which are superior to those of their individual counterparts. Recently, interfaced metal nanocatalysts on polymer/graphene composites with strong covalent bonds have provided superior characteristics, including size reduction, homogeneous distribution and excellent stability of metal nanocatalysts; this enables the uniform distribution of active sites and benefits superior NEGS performance associated with long durability. The significant efforts achieved in polymer/ graphene composites are elucidated as follows:

A Pd nanoparticles-decorated Nafion/graphene composite was synthesized via an in situ reduction process over the surface of GCE and utilized as an electrochemical probe for NEGS applications. The higher sulfate group density of Nafion in the Nafion/graphene composite electrostatically interacted with the positively charged $\mathrm{Pd}^{2+}$ ions, followed by chemical reduction with hydrazine hydrate to yield 30 to $40 \mathrm{~nm}$-sized Pd nanoparticles homogenously distributed over the Nafion/graphene matrix. Electrocatalytic glucose oxidation at the Pd/Nafion/ graphene composite was investigated with $\mathrm{CV}$ under alkaline conditions; an anodic peak was exhibited at $0.4 \mathrm{~V}$ vs. SCE, demonstrating the electrocatalytic glucose oxidation into glucolactone via $\mathrm{PdOH}$ species. It was also observed that the characteristic redox peak currents of the Pd/Nafion/graphene composite were higher than those of Nafion and Nafion/ graphene and Nafion/Pd composites owing to the combined properties of Pd, Nafion and graphene. The uniform and dense distribution of Pd nanoparticles over the Nafion/graphene matrix afforded numerous active sites for effectual glucose oxidation and exhibited considerable amperometric performance for glucose detection at $0.4 \mathrm{~V} v$ s. SCE under alkaline conditions. The reliability of these nanocomposites was verified by real sample glucose analysis in human blood serum, which ensured authentic glucose detection. ${ }^{138}$ Yang et al. developed a SPE decorated with a Ni/CS/rGO composite through electrodeposition for NEGS applications. The oxygen-labile functional groups of GO served as nucleation centres and electrostatically interacted with CS. Hydrophobic interactions also occurred between the glucosidic rings and acetyl groups of CS and the hydrophobic basal planes of GO. This was followed by a chelation effect between the $\mathrm{CS}$ and $\mathrm{Ni}^{2+}$ cations, which homogeneously anchored the 26 to $35 \mathrm{~nm}$ sized $\mathrm{Ni}$ nanoparticles over the $\mathrm{CS} / \mathrm{rGO}$ matrix. The as-prepared $\mathrm{Ni} / \mathrm{CS} / \mathrm{rGO} / \mathrm{SPE}$ was pretreated with $0.1 \mathrm{M} \mathrm{NaOH}$ with 40 consecutive cycles to reach a steady state peak current. The pretreated $\mathrm{Ni} / \mathrm{CS} / \mathrm{rGO} / \mathrm{SPE}$ was perfectly aligned with the microfluidic chamber enclosed in a polydimethylsiloxane (PDMS) chamber. For the sample 
injection, a $10 \mu \mathrm{L}$ microsyringei was connected to the inlet tube, and the tubular inlet was also joined to a pump and micropipettor/dropper. The samples were manually instilled at a gradual and constant rate or using a syringe pump, and the corresponding NEGS performance was monitored (Fig. 14). According to $\mathrm{CV}$ studies under alkaline conditions, the anodic peak was detected at $0.60 \mathrm{~V}$ vs. $\mathrm{Ag} / \mathrm{AgCl}$, signifying that the electrooxidation of glucose into glucolactone occurred at the $\mathrm{NiO}(\mathrm{OH})$ active centres. The $\mathrm{Ni} / \mathrm{CS} / \mathrm{rGO} / \mathrm{SPE}$ sensor provided a linearity and sensitivity of 0.2 to $9 \mathrm{mM}$ and $318.4 \mu \mathrm{AmM}$ $\mathrm{cm}^{-2}$, respectively, at $0.60 \mathrm{~V} v$ s. $\mathrm{Ag} / \mathrm{AgCl}$ toward glucose sensing, owing to the homogenous distribution and interconnected $\mathrm{Ni}$ nanoparticles in the CS/rGO matrix, which facilitated electron transfer kinetics with superior electrocatalytic activity. This composite exhibited enhanced specificity in human urine with an RSD of $8.7 \%$ when the SPE was integrated in a fabricated portable microfluidic device. ${ }^{139}$

The NEGS probe comprising Cu/polyacrylic acid (PAA)/ graphene ( $\mathrm{Cu} / \mathrm{PAA} /$ graphene) was achieved via a chemical reduction technique. PAA facilitated the growth of $\mathrm{Cu}$ nanoparticles with a higher dispersion on graphene; the crosslinking frameworks of PAA were incorporated into the stacked graphene layers through non-covalent functionalities, which stimulated mass transportation and enhanced the approachability of glucose toward the electroactive surface of the composite. In addition, PAA effectively prevented the agglomeration of $\mathrm{Cu}$ nanoparticles via the alignment of the carboxyl groups of PAA with the $\mathrm{Cu}$ nanoparticles. The fabricated $\mathrm{Cu}$ / $\mathrm{PAA} /$ graphene/GCE exhibited a linear range of 0.0003 to $0.6 \mathrm{mM}$ and a detection limit of $0.08 \mu \mathrm{M}$ at $0.4 \mathrm{~V} v$ s. SCE under alkaline conditions. The prepared material provided better results for glucose detection in human serum sample analysis, with an average RSD value of $3.24 \%$ in alkaline medium and excellent anti-interference properties. ${ }^{\mathbf{1 4 0}}$ The dendritic $\mathrm{Cu}-\mathrm{Co} /$ CS/rGO composite was developed over GCE via an electrodeposition technique. After an electrodeposition time of about $100 \mathrm{~s}$, flower-like Cu-Co structures were formed, and a further increase in the electrodeposition time up to $2600 \mathrm{~s}$ led to the growth of 3D dendritic structures containing holes and pores under alkaline conditions. Under alkaline conditions, $\mathrm{Cu}^{0} / \mathrm{Co}^{0}$ was transformed into $\mathrm{Cu}(\mathrm{II}) / \mathrm{Co}(\mathrm{II})$ and then further transformed into $\mathrm{Cu}(\mathrm{III}) / \mathrm{Co}(\mathrm{II})$; the analyte glucose was electrochemically oxidized at the $\mathrm{Cu}$ (III)/Co(II) active centres. In the bimetallic $\mathrm{Cu}^{-}$ Co nanostructures, Co enabled $\mathrm{Cu}$ to reach a higher oxidation state and lowered the peak potentials for the electrooxidation of glucose. Furthermore, the CS/rGO matrix facilitated the growth of 3D dendrites on the $\mathrm{Cu}-\mathrm{Co}$ structures via its large surface area and facilitated the electron transfer kinetics. $\mathrm{Cu}-\mathrm{Co} / \mathrm{CS} /$ $\mathrm{rGO} / \mathrm{GCE}$ provided greater amperometric response at $0.45 \mathrm{~V}$ vs. SCE toward glucose oxidation in comparison with $\mathrm{Cu}-\mathrm{Co} /$ GCE (Fig. 15a and b) owing to the combined properties of the individual components. Furthermore, the fabricated $\mathrm{Cu}-\mathrm{Co} / \mathrm{CS} /$ rGO/GCE displayed excellent selectivity toward glucose (Fig. 15c) and revealed an RSD value of $7.2 \%$ in fetal calf serum sample glucose analysis (Fig. 15d). ${ }^{\mathbf{1 4 1}} \mathrm{Ni}$ nanoparticles anchored over poly(3,4-ethylenedioxythiophene) (PEDOT)-doped rGO were fabricated by electropolymerization followed by
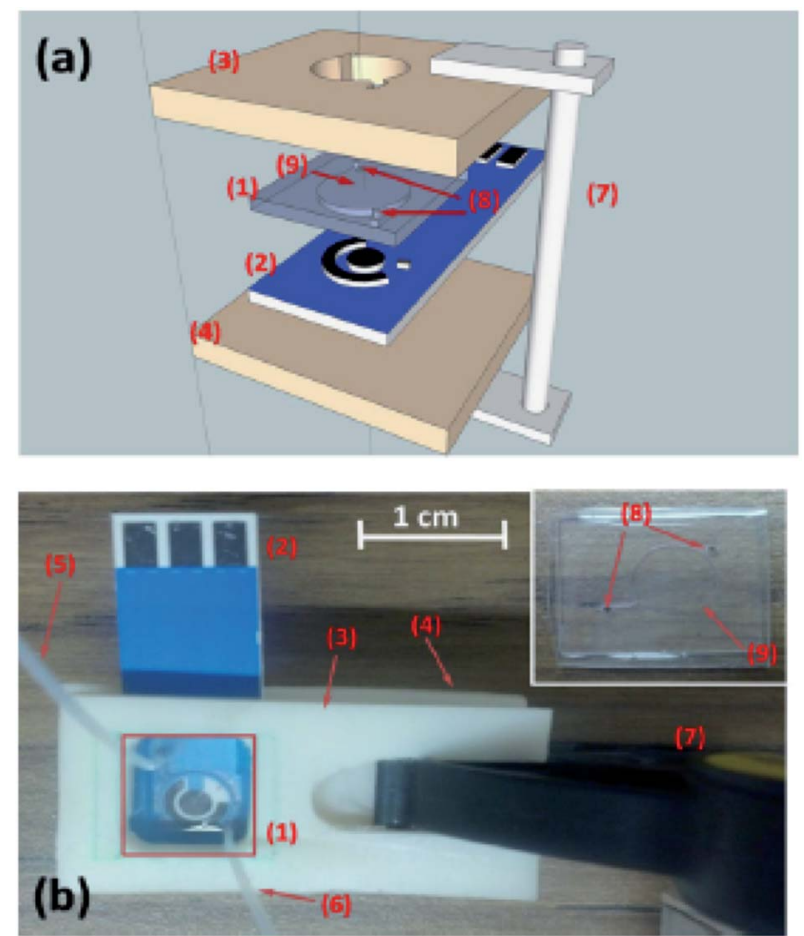

Fig. 14 (a) Schematic illustration of the setup of a device stack, (b) optical photograph of an as-constructed microfluidic NEGS device: (1) PDMS chamber (inset (b): enlarged view), (2) Ni/CS/rGO/SPE, (3) top and (4) bottom pieces of plastic device holder, (5) outlet tube, (6) inlet tube joined with sample injector, (7) microbar clamp/spreader, (8) holes for joining the outlet and inlet tubes, (9) circular chamber (reproduced with permission from Elsevier; Yang et al. 2013).

electrodeposition techniques; an assembly of Ni nanoparticles with average diameters of 50 to $100 \mathrm{~nm}$ was homogenously dispersed on the PEDOT/rGO matrix. CV studies revealed that the Ni/PEDOT/RGO/GCE probe electrooxidized glucose into glucolactone at $+0.5 \mathrm{~V} v$ s. SCE under alkaline conditions via the $\mathrm{NiO}(\mathrm{OH})$ electroactive species, whereas PEDOT/rGO/GCE did not induce glucose oxidation under similar conditions; this depicts the importance of the electroactive Ni nanoparticles. The PEDOT/rGO composite provided 3D conductive scaffolds for Ni deposition, which enhanced the electrocatalytic activity and high stability of the composite toward glucose oxidation. In addition, PEDOT offered a high charge injection limit, which enhanced the electrical conductive support behavior of graphene. In human serum sample analysis, the Ni/PEDOT/rGO sensor afforded a RSD value of $4.8 \%$, and its electrochemical activity was not influenced by the interference species. ${ }^{\mathbf{1 4 2}} \mathrm{A}$ polyvinyl acetate (PVA) electrode was modified with $\mathrm{MnO}_{2} / \mathrm{CuO}$ nanoparticles charged on $\mathrm{GO}\left(\mathrm{CuO} / \mathrm{MnO}_{2} / \mathrm{GO} / \mathrm{PVA}\right)$ using the molecular imprinting polymer (MIP) technique. The morphological characterizations revealed that $20 \mathrm{~nm}$-sized $\mathrm{CuO}$ nanoparticles were effectively embedded in $30 \mathrm{~nm}$-sized $\mathrm{MnO}_{2}$ nanorods, which were homogenously dispersed on the GO/PVA composite. The non-imprinted polymer electrode did not exhibit catalytic oxidation toward glucose under alkaline conditions. However, a $\mathrm{CuO} / \mathrm{MnO}_{2} / \mathrm{GO} / \mathrm{PVA}$ composite- 

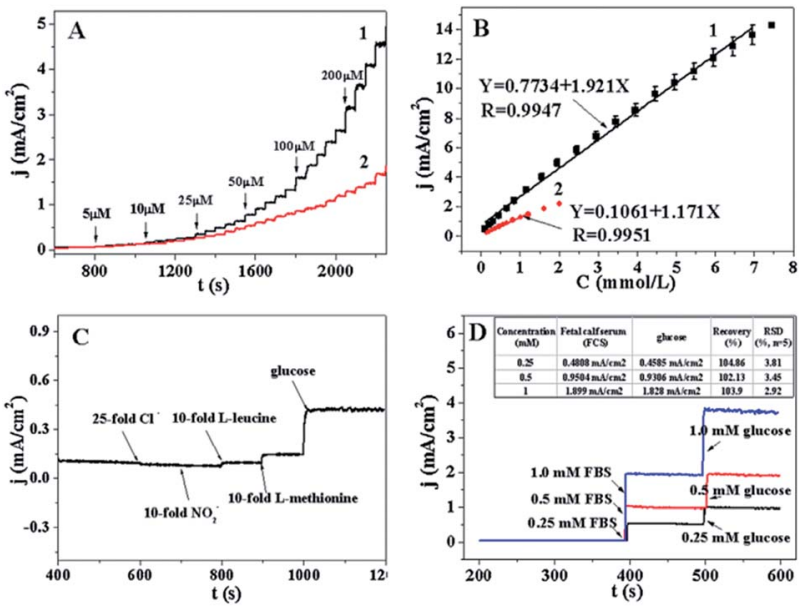

Fig. 15 (A) Typical steady-state responses of (1) Cu-Co/CS/rGO/GCE and (2) $\mathrm{Cu}-\mathrm{Co} N S s / G C E$ to the consecutive injection of glucose in $0.1 \mathrm{M} \mathrm{NaOH}$ solution and (B) correlating calibration plots, (C) chronoamperometric responses for glucose and interference of $\mathrm{Cl}^{-}, \mathrm{NO}_{2}{ }^{-}$, L-leucine and L-methionine and (D) detection of simulated practical samples at an applied potential of $0.45 \mathrm{~V}$ vs. SCE in $0.1 \mathrm{M} \mathrm{NaOH}$ solution (reproduced with permission from Elsevier; Wang et al. 2014).

modified MIP electrode exhibited electrocatalytic glucose oxidation at $0.4 \mathrm{~V} v s$. $\mathrm{Ag} / \mathrm{Ag} \mathrm{Cl}$ under alkaline conditions owing to the number of recognition sites afforded by the MIP for the target molecule to rebind; this demonstrated the effects of the electrochemical probe on the target glucose molecule. The fabricated $\mathrm{CuO} / \mathrm{MnO}_{2} / \mathrm{GO} / \mathrm{PVA}$ MIP sensor afforded a strong platform for glucose sensing, with a linearity of 0.5 to $4.4 \mathrm{mM}$ and a detection limit of $53 \mu \mathrm{M} .{ }^{143}$ The research efforts on $\mathrm{CuO} /$ $\mathrm{MnO}_{2} / \mathrm{GO} / \mathrm{PVA}$ have also provided significant improvements in NEGS performance. ${ }^{144}$

The integration of polymers with graphene in advanced multifunctional composites provides advantageous features, including high tensile strength, exceptional specific modulus, low cost, scalability, good dispersibility, easier synthesis protocols, biocompatibility, and high charge injection limit, with excellent preservation of the native structure and properties of graphene. The individual limitations of polymers, including swelling, shrinkage, inferior stability and sluggish electrokinetics, and the restacking issues of graphene sheets were effectively addressed with the above integration, which improved the redox behaviour of the resulting composites toward glucose detection. However, the low intrinsic reactivity of graphene-based platforms limits its interfacial interactions with the polymer; this affects the load transfer across the interface, leading to decreased NEGS performance. The stimulation of covalent functional bonds disrupted the $\pi$ conjugation of graphene, which leads to a negative impact on electrical properties corresponding composites. The non-covalent and covalent functionalization of polymers on graphene stimulated the compatibilizer-polymer interaction, which lead to increased electrical and mechanical properties. The role of the interface between the graphene with the non-covalent functionalization and the polymeric matrix in simultaneously enhancing the electrical and mechanical properties of composite is not as yet understood. Hence, the covalent or supramolecular interaction between graphene and the polymer is essential to tune their interface interactions to achieve a highly stable dispersion, which may lead to enhanced NEGS performance.

\subsection{Heteroatom-doped graphene composites}

Heteroatom-doped graphene has been introduced to the field of NEGS owing to its p-type semiconductor behaviour; the heteroatoms doping on graphene facilitates edge exposure and provides considerable electron transfer channels, which collectively reduces the internal resistance of a system. As the heteroatoms in graphene alone are not influential enough for the effectual electrooxidation of glucose, numerous metal and metal oxide nanoparticles have been anchored on active graphene, and the influences of the prepared composites toward NEGS performance have been categorized as follows: nitrogendoped graphene with $\mathrm{Cu}$ nanoparticles $(\mathrm{Cu} / \mathrm{NG})$ were prepared by a facile thermal treatment, and the $\mathrm{N}$ doping level in $\mathrm{Cu} / \mathrm{NG}$ was found to be $\sim 15 \%$. The as-prepared NG composite exhibited various forms of $\mathrm{N}$, including pyridinic, amino and pyrrolic $\mathrm{N}$, and demonstrated high charge mobility and enhanced carbon catalytic activity. In addition, the $\mathrm{C}-\mathrm{N}$ microenvironment provided a large active surface area to the $\mathrm{Cu} / \mathrm{NG}$ composite by enhancing the volume ratio of the active surface groups as well as the electrical conductivity and biocompatibility. Morphological studies revealed that cubic $\mathrm{Cu}$ nanoparticles 80 to $120 \mathrm{~nm}$ in size were homogeneously assembled on the crumpled multilayer surface of the NG structure. CV studies revealed that the glucose oxidation occurred at $+0.5 \mathrm{~V} v s$. SCE for $\mathrm{Cu} / \mathrm{NG} / \mathrm{GCE}$ owing to the engagement of $\mathrm{Cu}(\mathrm{II}) / \mathrm{Cu}(\mathrm{III})$ surface species; the corresponding electrochemical response was 23 fold higher than that of $\mathrm{Cu} / \mathrm{GCE}$, indicating the enhanced electron transfer chain and excellent catalytic activity of NG and $\mathrm{Cu}$ nanoparticles, respectively. $\mathrm{Cu} / \mathrm{NG} / \mathrm{GCE}$ exhibited rapid amperometric performance in glucose detection at $+0.5 \mathrm{~V}$ vs. SCE, and its analytical reliability was demonstrated in human blood serum analysis. ${ }^{144}$ A Cu-NiO nanoparticlesdecorated NG composite was prepared by a one-pot solvothermal technique, and the hierarchical characterization of the as-prepared nanocomposite showed that $\mathrm{Cu}-\mathrm{NiO}$ with a mean diameter of $5.6 \mathrm{~nm}$ was uniformly dispersed on the rippled, crumpled paper-like surface of the NG sheets, which comprised $6.8 \% \mathrm{~N}$. The electrochemical activities of $\mathrm{Cu}-\mathrm{NiO} /$ NG/ITO were studied by CV, showing a significant glucose oxidation peak at $+0.58 \mathrm{~V} v$ s. $\mathrm{Ag} / \mathrm{AgCl}$ under alkaline conditions; this indicates the effective conjugation of bimetallic $\mathrm{Cu}-\mathrm{NiO}$ and NG. The fabricated sensor exhibited rapid amperometric response toward the electrochemical oxidation of glucose in alkaline medium. The structural change of graphene with $\mathrm{N}$ doping increased the conductivity by the strong electrostatic interaction between $\mathrm{Cu}-\mathrm{NiO}$ and $\mathrm{N}$; this accounts for the prominent catalytic property of the composite, which collectively enhanced the glucose oxidation. ${ }^{145} \quad \mathrm{Mn}_{3} \mathrm{O}_{4} / \mathrm{NG}$ was prepared by a hydrothermal technique, and its stability was enhanced by the addition of a capping agent, ethylene diamine, for NEGS applications. Morphological studies demonstrated 
that $\mathrm{Mn}_{3} \mathrm{O}_{4}$ nanoparticles were homogenously dispersed on NG with sizes of 50 to $80 \mathrm{~nm}$, while a $9.86 \%$ atomic ratio of nitrogen was doped on graphene. The electrocatalytic activity of $\mathrm{Mn}_{3} \mathrm{O}_{4} /$ NG/CPE was appraised by $\mathrm{CV}$; it exhibited a greater faradic current owing to superior electrocatalytic activity upon the addition of glucose under alkaline conditions, where glucose oxidation was achieved by the $\mathrm{MnO}_{2}$ species via the gluconolactone intermediate. Moreover, the observed electrochemical activity can be ascribed to the well-distributed and extended number of catalytic active sites and the high loading amount of $\mathrm{Mn}_{3} \mathrm{O}_{4}$ nanoparticles, which facilitated the electrical network via anchoring on the surface of doped graphene; $\mathrm{Mn}_{3} \mathrm{O}_{4} / \mathrm{NG} /$ CPE exhibited a 10 fold higher electrocatalytic glucose oxidation than bare CPE. The high electrocatalytic action of glucose oxidation through $\mathrm{Mn}-\mathrm{N}$ interactions with the $\mathrm{Mn}_{3} \mathrm{O}_{4} / \mathrm{NG}$ composite provided a considerable amperometric response. The fabricated sensor also showed excellent reliability toward human urine sample analysis in glucose detection by avoiding the poisoning effect of chloride ions. ${ }^{\mathbf{1 4 6}}$ An electrochemical probe with $\mathrm{Mn}_{3} \mathrm{O}_{4}$ nanoparticles-functionalized N-rGO $\left(\mathrm{Mn}_{3} \mathrm{O}_{4} /\right.$ $\mathrm{N}$-rGO) demonstrated potential applications in the field of NEGS; the electrocatalyst was prepared by a simple hydrothermal technique. Morphological studies clearly depicted the homogeneous distribution of 30 to $80 \mathrm{~nm}$-sized $\mathrm{Mn}_{3} \mathrm{O}_{4}$ nanoparticles on the surface of transparent N-rGO sheets, and the formation of $\mathrm{Mn}_{3} \mathrm{O}_{4}$ structures on $\mathrm{N}$-rGO was confirmed by the splitting width of $11.50 \mathrm{eV}$, obtained via XPS. In electrocatalytic glucose detection, the glucose oxidation peak current of $\mathrm{Mn}_{3} \mathrm{O}_{4}$ / $\mathrm{N}-\mathrm{rGO} / \mathrm{CPE}$ was enhanced by 3.5 times over the $\mathrm{Mn}_{3} \mathrm{O}_{4} / \mathrm{CPE}$; this is attributed to the availability of numerous active sites with high loading amounts of $\mathrm{Mn}_{3} \mathrm{O}_{4}$ nanoparticles and to the increment in the interaction between graphene and the nanoparticles through N-doping. This hybrid showed good amperometric response at $+0.7 \mathrm{~V}$ vs. $\mathrm{Ag} / \mathrm{AgCl}$, and the developed composite provided enhanced selectivity ( 20 fold that of GCE) and rapid human blood sample analysis owing to the strong electrostatic interaction between $\mathrm{N}$ and $\mathrm{Mn}^{\mathbf{1 4 7}} \mathrm{A} \quad \mathrm{CuO}$ nanoparticles-anchored sulfur doped graphene nanocomposite (CuO/SG) was developed by a microwave-aided method; $2.17 \% \mathrm{~S}$ was effectively doped in the graphene matrix as evidenced by XPS, which demonstrated that microwave-assisted technique is sufficient to introduce $S$ content in graphene. As the electronegativity of $S$ atom (2.58) is similar to that of carbon (2.55), the effectual doping of $\mathrm{S}$ on graphene was achieved with high spin density; also, SG provided good semiconducting properties, which enhanced the application of this material in NEGS. A slightly higher shift was observed for the $\mathrm{Cu} 2 \mathrm{p}^{3 / 2}$ and $\mathrm{Cu} 2 \mathrm{p}^{1 / 2}$ peaks of $\mathrm{CuO} / \mathrm{SG}$ in comparison with $\mathrm{CuO} / \mathrm{rGO}$, which revealed that the transfer of electrons occurred from $\mathrm{CuO}$ to $\mathrm{SG}$ and indicated the generation of possible $\mathrm{Cu}-\mathrm{S}$ interactions between the $\mathrm{CuO}$ nanoparticles and SG. The strong CuO-SG bonds resulted in 10 to $16 \mathrm{~nm}$-sized $\mathrm{CuO}$ nanoparticles on the SG matrix, which were smaller than the $\mathrm{CuO}$ nanoparticles anchored on the rGO matrix (18 to $25 \mathrm{~nm}$ ). This was also reflected in the large surface area of the $\mathrm{CuO} / \mathrm{SG}$ composite $(247.8$ $\left.\mathrm{m}^{2} \mathrm{~g}^{-1}\right)$ in comparison with $\mathrm{CuO} / \mathrm{rGO}\left(216.3 \mathrm{~m}^{2} \mathrm{~g}^{-1}\right)$ and the $\mathrm{CuO}$ nanoparticles $\left(\begin{array}{lll}64.8 & \mathrm{~m}^{2} & \mathrm{~g}^{-1}\end{array}\right)$. The electrochemical characterization in alkaline medium of the as-fabricated $\mathrm{CuO} /$ $\mathrm{SG} / \mathrm{GCE}$ sensor showed a response at $\sim+0.5 \mathrm{~V} v s$. Ag/AgCl electrode in glucose detection, owing to the involvement of the $\mathrm{Cu}(\mathrm{II}) / \mathrm{Cu}(\mathrm{III})$ active species facilitated glucose detection. A stepwise increase in amperometric analysis revealed that the $\mathrm{CuO} /$ SG nanocomposite exhibited a linear range of 0.1 to $10.5 \mathrm{mM}$, a sensitivity of $1298.6 \mu \mathrm{A} \mathrm{mM} \mathrm{m}^{-1} \mathrm{~cm}^{-2}$, a low detection limit of $0.08 \mu \mathrm{M}$ and a rapid detection time of $2 \mathrm{~s}$ toward glucose detection at $+0.5 \mathrm{~V} v s$. $\mathrm{Ag} / \mathrm{AgCl}$ owing to the enhanced electron donor ability of the $\mathrm{Cu}-\mathrm{S}$ interaction. ${ }^{148}$ The significant electrochemical achievements in polymer/graphene and metal nanoparticles/heteroatom-doped graphene-equipped NEGS are provided in Table $4 .^{\mathbf{1 3 8 - 1 4 8}}$ The significant constraints associated with metal nanostructures/graphene composites were addressed by heteroatom-doped graphene, which offers numerous superior advantages, including elevated electrondonor properties, biocompatibility, binding ability, hydrophilicity, stability and electrical conductivity; this influenced the durability of the NEGS. However, a decrease in carrier mobility is observed for heteroatom-doped graphene, achieved via the alteration of the native electronic structure, and the altered physical properties of graphene with the conversion of $\mathrm{sp}^{2}$ carbons into $\mathrm{sp}^{3}$ hybridized carbons and the loss of conjugation limit its electrocatalytic activities toward glucose oxidation. Furthermore, the exact mechanism involved in the electrooxidation of glucose at heteroatom-doped graphene is yet to be clearly understood, which limits the fundamental understanding of its applications in NEGS. Effectual pathways designed based on the above perspectives will lead to a fundamental breakthrough in the development of highly durable NEGS.

From the aforementioned research efforts, it is clear that NEGS equipped with graphene-based platforms not only address the limitations of EGS, including the inevitable enzymerelated constraints and minimal durability, but also outperform the unique advantages of EGS from the perspectives of high sensitivity and selectivity. The robust framework and improved electrochemical and physiochemical properties of graphene platforms improve the interfacial properties between the current collector and glucose, which collectively enhances their excellent sensitivity and selectivity toward glucose oxidation and has greatly increased the commercialization opportunities of graphene platform-based NEGS.

\section{Future perspectives}

Although considerable research progress has been made on graphene platform-based NEGS, the development and commercialization of miniaturized portable NEGS may materialize with the design and development of effective graphenebased platforms with multifunctional reactivity and affordable cost; the following research directions will be useful in achieving state-of-the art graphene platforms.

(i) Owing to the exceptional electrochemical performance of graphene platform-based NEGS, the requirements of the developed system will surpass those of conventional systems; this will necessitate high-volume production of graphene at an 


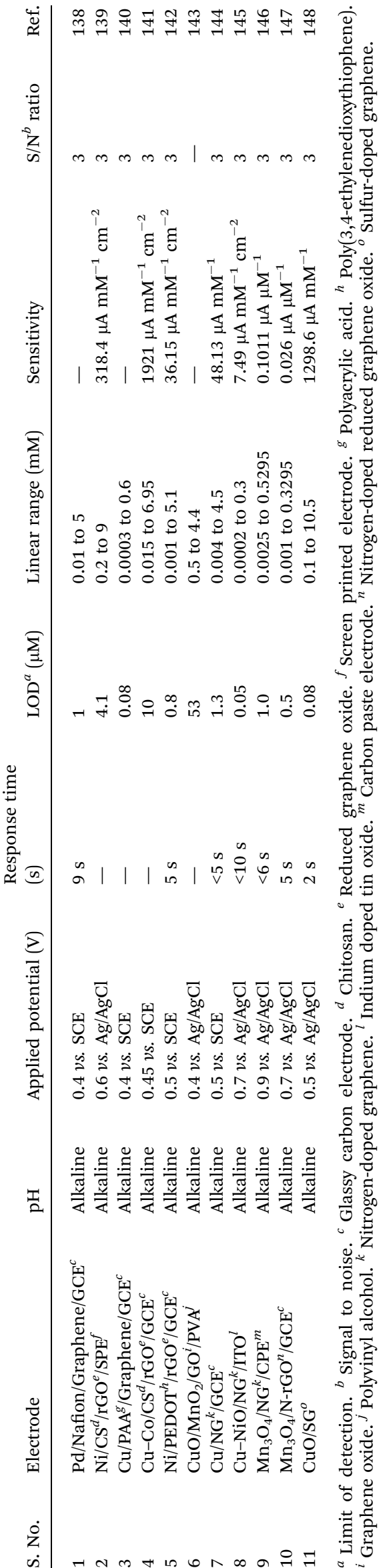

affordable cost for greatly successful commercialization. The preparation of defect-free pristine graphene from the exfoliation of graphite using the Scotch tape method does not satisfy the prerequisites of large-scale device fabrication. Although this was addressed by the chemical exfoliation of graphite and its chemical and thermal reduction, the resulting graphene platforms exhibited lower reactivity along with inferior electrical and mechanical properties. Hence, the scalable CVD technique must be extensively exploited to tune and deposit various layers of graphene platforms on different substrate materials which can be directly used for the fabrication of flexible, lightweight, miniaturized and portable NEGS devices that will eventually decrease the overall cost of such systems.

(ii) The electrocatalytic activities of graphene-based platforms are purely dependent upon the structural disorder, defects, conjugated domains and functional groups of graphene. However, their influences on NEGS performance are not yet understood and should be addressed in detail.

(iii) The number of sequential steps involved in the development of conventional graphene-based NEGS, including laborious electrode cleaning and polishing, binder selection and utilization, preparation of catalyst slurries and catalyst loading processes, have collectively increased the time and cost consumption processes of NEGS. Though a few attempts have been achieved on stimulating a good connection between the catalyst and current collector with the aid of binder materials, the high contact resistance still remains a crucial challenge for the high performance NEGS. This could be effectively addressed with the development of binder-free and free-standing nanofiber membranes, foams, gels and foil-based electrodes, by which the preparation and modification of graphene-based electrode materials could be simultaneously achieved.

(iv) Most research efforts on NEGS have been achieved only on graphene nanosheets, which limits the fundamental understanding of the influence of the host matrix morphology on glucose quantification and its interaction with guest materials. A proper focus on the development of graphene matrices with different morphologies, including spheres, dots, ribbons, rods and tubes, and a detailed study of the tunable pore properties to achieve an extended number of active sites and large volumetric surface areas will provide a positive impact on the development of efficient NEGS.

(v) Although the agglomeration and swelling behaviors of metal nanoparticles and conductive polymers can be controlled by the active graphene support, the durability of NEGS could be further improved with heteroatom-doped graphene, which provides stability to catalysts. However, the active sites implanted in heteroatom-doped graphene do not effectively influence the effectual electrooxidation of glucose. To understand the exact mechanism, proper efforts should be devoted to increase the oxidation active sites in heteroatom-doped graphene to increase glucose quantification. If the doping of heteroatoms on graphene can be achieved with sustainable biomass materials, the environmentally benign characteristics of NEGS can be further improved.

(vi) The fundamental understanding of glucose oxidation mechanisms and their connectivity with graphene-based 
platforms could be clearly visualized by establishing theoretical models, including molecular, atomic and electronic models, and relevant experimental approaches.

(vii) The graphene platform-based NEGS technology must be extended from disposable strips to microfluidic chips for the empowerment of next-generation electronic devices. Proper implementation of the above technology will definitely lead to wearable and implantable NEGS devices, which will effectively avoid the limitations of clinical treatment of patients.

\section{Conclusion}

The immense demand for the effective management of diabetes necessitates the design and development of advanced NEGS equipped with graphene-based platforms; the enormous research effort devoted to this area greatly reflects the clinical importance of this field. Although the rich chemistry of graphene-based platforms offers significant improvements in NEGS, significant further efforts are highly essential to (i) understand the interaction mechanisms between graphene platforms and the glucose and electrooxidation mechanisms of glucose at graphene-based platforms, (ii) evaluate the factors that improve the sensitivity and selectivity of NEGS, (iii) improve the durability and consistency of NEGS, (iv) actualize real-time applications and (v) commercialize miniaturized digital sensors. On this basis, the required parameters of NEGS catalysts and the influences of graphene on each parameter have been well described in this article. The research efforts devoted to NEGS equipped with graphene-based platforms have been segmented into six categories, including noble metal nanoparticles/graphene, non-noble metal nanoparticles/ graphene, non-noble metal oxide nanoparticles/graphene, alloy/hybrid metal nanoparticles/graphene, polymers/ graphene and metal nanoparticles/heteroatom-doped graphene. This brief study of the unique properties of graphenebased NEGS and explanation of the data from previous research endeavors provides a fundamental understanding of the substantial development of glucose monitoring systems. Despite the impressive research progress, the promise of effective glucose monitoring has not been fulfilled, and some challenges and obstacles remain related to the achievement of reliable and continuous glucose monitoring. However, the ultimate implementation of novel NEGS devices is not only dependent upon scientific issues but also relies upon commercial and legal considerations, which necessitates momentous efforts to couple basic science with advance technological features with a view to future directions. Hence, this article may be the only review that serves as a building block for the basic understanding of tuning the surface structures of graphene platforms for the commercialization of NEGS.

\section{Acknowledgements}

This study was supported by the Science and Engineering Research Board, New Delhi, India, Major Project Grant No. EMR/2015/000912.

\section{References}

1 R. J. Shaw, Curr. Opin. Cell Biol., 2006, 18, 598-608.

2 T. Ramasarma and M. Rafi, Indian J. Exp. Biol., 2016, 54, 8399.

3 M. M. A. Andany, M. G. Lucan, C. D. Garcia, C. F. Fernandeza and E. A. Rodriguez, BBA Clinical, 2016, 5, 85-100.

4 S. L. Aronoff, Diabetes Spectrum, 2008, 17, 3.

5 P. Reichard, M. Pihl, U. Rosenqvist and J. Sule, Diabetologia, 1996, 39, 1483-1488.

6 A. A. Onitilo, J. M. Engel, I. Glurich, R. V. Stankowski, G. M. Williams and S. A. Do, Canc. Causes Contr., 2012, 23, 967-981.

7 A. A. Onitilo, J. M. Engel, I. Glurich, R. V. Stankowski, G. M. Williams and S. A. Do, Canc. Causes Contr., 2012, 23, 991-1008.

8 T. A. Chowdhury, S. Ahmad and B. J. Boucher, Journal of Diabetes and its Complications, 2013, 27, 184-190.

9 B. Luna and M. N. Feinglos, JAMA, 2001, 16, 1945-1948.

10 D. R. Whiting, L. Guariguata, C. Weil and J. Shaw, Diabetes Res. Clin. Pract., 2011, 94, 311-321.

11 G. Atshushi, Biomed. Lett., 2013, 347, f4533.

12 I. Chinedu, F. O. Uhegbu, I. Chukwuechefulam, I. Nkeiruka, O. Kelech and I. Chibuike, Indian J. Med. Microbiol., 2013, 1, 7-12.

13 A. Pfützner, A. Caduff, M. Larbig, T. Schrepfer and T. Forst, Diabetes Technol. Ther., 2004, 6, 435-441.

14 Y. C. Shen, A. G. Davies, E. H. Linfield, P. Taday and D. D. Arnone, Phys. Med. Biol., 2003, 48, 2023-2032.

15 T. Kubiak, B. Wörle, B. Kuhr, I. Nied, G. Gläsner and N. Hermanns, Diabetes Technol. Ther., 2006, 8, 570-575.

16 S. Mansouri and J. S. Schultz, Biotechnology, 1984, 2, 885890.

17 G. Rao, P. Glikfeld and R. H. Guy, Pharm. Res., 1993, 10, 1751-1755.

18 J. Kost, S. Mitragotri, R. A. Gabbay, M. Pishko and R. Langer, Nat. Med., 2000, 6, 347-350.

19 H. Chuang, E. Taylor and T. W. Davidson, Diabetes Technol. Ther., 2004, 6, 21-30.

20 J. Wang, Chem. Rev., 2008, 108, 814-825.

21 M. García, P. Batalla and A. Escarpa, Trends Anal. Chem., 2014, 57, 6-22.

22 W. Franke and M. Deffner, Justus Liebigs Ann. Chem., 1939, 541, 117-150.

23 J. Ding, W. Sun, G. Wei and Z. Su, RSC Adv., 2015, 5, 3533835345.

24 A. Heller and B. Feldman, Chem. Rev., 2008, 108, 2482-2505.

25 S. Park, H. Boo and T. D. Chung, Anal. Chim. Acta, 2006, 556, 46-57.

26 G. Ron, Angew. Chem., 2008, 120, 1700-1703.

27 Y. Umasankar and S. M. Chen, Sensors, 2008, 8, 290-313.

28 B. Perez-Lopez and A. Merkoçi, Microchim. Acta, 2012, 179, 1-16.

29 L. Q. Rong, C. Yang, Q. Y. Qian and X. H. Xia, Talanta, 2007, 72, 819-824. 
30 K. E. Toghill and R. G. Compton, Int. J. Electrochem. Sci., 2010, 5, 1246-1301.

31 A. Martín, J. H. Ferrer, M. T. Martínez and A. Escarpa, Electrochim. Acta, 2015, 172, 2-6.

32 D. Pletcher, J. Appl. Electrochem., 1984, 14, 403-415.

33 L. D. Burke, Electrochim. Acta, 1994, 39, 1841-1848.

34 G. Wang, X. Lu, T. Zhai, Y. Ling, H. Wang, Y. Tong and Y. Li, Nanoscale, 2012, 4, 3123-3127.

35 J. Salamon, Y. Sathishkumar, K. Ramachandran, Y. S. Lee, D. J. Yoon, A. R. Kim and G. Gnana kumar, Biosens. Bioelectron., 2015, 64, 269-276.

36 A. Martin, J. H. Ferrer, L. Vazquez, M. Teresa and A. Escarpa, RSC Adv., 2014, 4, 132-139.

37 G. G. Kumar, Z. Awan, K. S. Nahm and J. S. Xavier, Biosens. Bioelectron., 2014, 53, 528-534.

38 X. Y. Lang, H. Y. Fu, C. Hou, G. F. Han, P. Yang, Y. B. Liu and Q. Jiang, Nat. Commun., 2012, 3169.

39 M. F. E. Kady, V. Strong, S. Dubin and R. B. Kaner, Science, 2012, 335, 1326-1330.

40 T. Alizadeh and S. Mirzagholipur, Sens. Actuators, B, 2014, 198, 438-447.

41 A. C. Ferrari, F. Bonaccorso, V. Falko, K. S. Novoselov, S. Roche, P. Bøggild, S. Borini, F. H. L. Koppens, V. Palermo, N. Pugno, J. A. Garrido, R. Sordan, A. Bianco, L. Ballerini, M. Prato, E. Lidorikis, J. Kivioja, C. Marinelli, T. Ryhänen, A. Morpurgo, J. N. Coleman, V. Nicolosi, L. Colombo, A. Fert, M. G. Hernandez, A. Bachtold, G. F. Schneider, F. Guinea, C. Dekker, M. Barbone, Z. Sun, C. Galiotis, A. N. Grigorenko, G. Konstantatos, A. Kis, M. Katsnelson, L. Vandersypen, A. Loiseau, V. Morandi, D. Neumaier, E. Treossi, V. Pellegrini, M. Polini, A. Tredicucci, G. M. Williams, B. H. Hong, J. H. Ahn, J. M. Kim, H. Zirath, B. J. van Wees, H. van der Zant, L. Occhipinti, A. D. Matteo, I. A. Kinloch, T. Seyller, E. Quesnel, X. Feng, K. Teo, N. Rupesinghe, P. Hakonen, S. R. T. Neil, Q. Tannock, T. Löfwander and J. Kinaret, Nanoscale, 2015, 7, 4598-4810.

42 V. Singh, D. Joung, L. Zhai, S. Das and S. I. Khondaker, Prog. Mater. Sci., 2011, 56, 1178-1271.

43 A. Martín and A. Escarpa, Trends Anal. Chem., 2014, 56, 1326.

44 S. Brahim, D. Narinesingh and A. G. Elie, Anal. Chim. Acta, 2001, 448, 27-36.

45 X. Yu, W. Zhang, P. Zhang and Z. Su, Biosens. Bioelectron., 2017, 89, 72-84.

46 A. M. Pinto, I. C. Gonçalves and F. D. Magalhaes, Colloids Surf., B, 2013, 111, 188-202.

47 X. Zhao, P. Zhang, Y. Chen, Z. Su and G. Wei, Nanoscale, 2015, 7, 5080-5093.

48 Z. Tang, H. Wu, J. R. Cort, G. W. Buchko, Y. Zhang, Y. Shao, I. A. Aksay, J. Liu and Y. Lin, Small, 2010, 6, 1205-1209.

49 C. Shan, H. Yang, D. Han, Q. Zhang, A. Ivaska and L. Niu, Langmuir, 2009, 25, 12030-12033.

50 J. T. Robinson, S. M. Tabakman, Y. Liang, H. Wang, H. S. Casalongue, D. Vinh and H. Dai, J. Am. Chem. Soc., 2011, 133, 6825-6831.
51 P. Zhang, Y. Huang, X. Lu, S. Zhang, J. Li, G. Wei and Z. Su, Langmuir, 2014, 30, 8980-8989.

52 D. Rana and T. Matsuura, Chem. Rev., 2010, 110, 2448-2471.

53 L. Yu, Y. Zhang, B. Zhang, J. Liu, H. Zhang and C. Song, J. Membr. Sci., 2013, 7, 42.

54 M. Ranjani, Y. Sathishkumar, Y. S. Lee, D. J. Yoo, A. R. Kim and G. G. Kumar, RSC Adv., 2015, 5, 57804-57814.

55 A. Safavi, N. Malekia and E. Farjami, Biosens. Bioelectron., 2009, 24, 1655-1660.

56 P. Si, Y. Huang, T. Wang and J. Ma, RSC Adv., 2013, 3, 34873502.

57 A. Primo, P. Atienzar, E. Sanchez, J. M. Delgadob and H. Garcia, Chem. Commun., 2012, 48, 9254-9256.

58 O. Akhavan and E. Ghaderi, Carbon, 2012, 50, 1853-1860.

59 A. K. Geim and K. S. Novoselov, Nat. Mater., 2007, 6, 183191.

60 A. K. Geim, Science, 2009, 324, 1530.

61 F. Schedin, A. K. Geim, S. V. Morzov, E. W. Hill, P. Blake, M. I. Katsnelson and K. S. Novoselov, Nat. Mater., 2007, 6, 652-655.

62 C. Shan, H. Yang, J. Song, D. Han, A. Ivaska and L. Niu, Anal. Chem., 2009, 81, 2378-2382.

63 M. Zhou, Y. Zhai and S. Dong, Anal. Chem., 2009, 81, 56035613.

64 A. K. Geim. Nobel Lecture, December 8, 2010.

65 Y. Wang, Y. Shao, D. W. Matson, J. Li and Y. Lin, ACS Nano, 2010, 1790-1798.

66 C. Shana, H. Yanga, D. Hana, Q. Zhanga, A. Ivaskab and L. Niua, Biosens. Bioelectron., 2010, 25, 1070-1074.

67 H. Gao, F. Xiao, C. B. Ching and H. Duan, ACS Appl. Mater. Interfaces, 2011, 3, 3049-3057.

68 X. C. Dong, H. Xu, X. W. Wang, Y. X. Huang, M. B. C. Park, H. Zhang, L. H. Wang, W. Huang and P. Chen, ACS Nano, 2012, 6, 3206-3213.

69 J. Yang, J. H. Yu, J. Rudi Strickler, W. J. Chang and S. Gunasekaran, Biosens. Bioelectron., 2013, 47, 530-538.

70 N. S. Ismail, Q. H. Le, H. Yoshikawa, M. Saito and E. Tamiya, Electrochim. Acta, 2014, 146, 98-105.

71 I. Shackery, U. Patil, A. Pezeshki, N. M. Shinde, S. Kang, S. Im and S. C. Jun, Electrochim. Acta, 2016, 191, 954-961.

72 F. Y. Kong, X. R. Li, W. W. Zhao, J. J. Xu and H. Y. Chen, Electrochem. Commun., 2012, 14, 59-62.

73 Y. Liu, Y. Dong, C. X. Guo, Z. Cui, L. Zheng and C. M. Li, Electroanalysis, 2012, 24, 2348-2353.

74 Q. Wang, X. Q. Cui, J. Chen, X. Zheng, C. Liu, T. Xue, H. Wang, Z. Jinz, L. Qiao and W. Zheng, RSC Adv., 2012, 2, 6245-6249.

75 J. W. Wu, C. H. Wanga, Y. C. Wanga and J. K. Chang, Biosens. Bioelectron., 2013, 46, 30-36.

76 G. H. Wu, X. H. Song, Y. F. Wua, X. M. Chen, F. Luo and X. Chen, Talanta, 2013, 105, 379-385.

77 L. Ruiyi, Z. Juanjuan, W. Zhouping, L. Zaijun, L. Junkang, G. Zhiguo and W. Guangli, Sens. Actuators, B, 2015, 208, 421-428.

78 A. C. Joshi, G. B. Markad and S. K. Haram, Electrochim. Acta, 2015, 161, 108-114. 
79 L. T. Hoa, K. G. Sun and S. H. Hur, Sens. Actuators, B, 2015, 210, 618-623.

80 H. Shu, G. Chang, J. Su, L. Cao, Q. Huang, Y. Zhang, T. Xia and Y. He, Sens. Actuators, B, 2015, 220, 331-339.

81 Y. Luo, F. Y. Kong, C. Li, J. J. Shi, W. X. Lv and W. Wang, Sens. Actuators, B, 2016, 234, 625-632.

82 T. D. Thanh, J. Balamurugan, J. Y. Hwang, N. H. Kim and J. H. Lee, Carbon, 2016, 98, 90-98.

83 Z. Fan, B. Liu, X. Liu, Z. Li, H. Wang, S. Yanga and J. Wang, Electrochim. Acta, 2013, 109, 602-608.

84 B. Wang, S. Li, J. Liu and M. Yu, Mater. Res. Bull., 2014, 49, 521-524.

85 B. Wang, Y. Wu, Y. Chena, B. Wenga and C. Lia, Sens. Actuators, B, 2017, 238, 802-808.

86 Z. Shen, W. Gao, P. Li, X. Wang, Q. Zheng, H. Wu, Y. Mab, W. Guan, S. Wu, Y. Yua and K. Ding, Talanta, 2016, 159, 194-199.

87 F. Tehrani and B. Bavarian, Sci. Rep., 2016, 6, 27975-27984.

88 Q. Wang, Q. Wang, M. Li, S. Szunerits and R. Boukherroub, RSC Adv., 2015, 5, 1586.

89 Z. Wang, Y. Hu, W. Yang, M. Zhou and X. Hu, Sensors, 2012, 12, 4860-4869.

90 M. Liu, R. Liu and W. Chen, Biosens. Bioelectron., 2013, 45, 206-212.

91 X. Wang, E. Liu and X. Zhang, Electrochim. Acta, 2014, 130, $253-260$.

92 Y. Zhao, X. Bo and L. Guo, Electrochim. Acta, 2015, 176, 1272-1279.

93 D. Ye, G. Liang, H. Li, J. Luo, S. Zhang, H. Chen and J. Kong, Talanta, 2013, 116, 223-230.

94 J. Zheng, W. Zhang, Z. Lin, C. Wei, W. Yang, P. Dong, Y. Yan and S. Hu, J. Mater. Chem. B, 2016, 4, 1247.

95 L. P. Mei, P. Song, J. J. Feng, J. H. Shen, W. Wang, A. J. Wang and X. Weng, Microchim. Acta, 2015, 182, 1701-1708.

96 X. Feng, C. Guo, L. Mao, J. Ning and Y. Hu, J. Am. Ceram. Soc., 2014, 97, 811-815.

97 H. Y. Yu, M. Q. Xu, S. H. Yu and G. C. Zhao, Int. J. Electrochem. Sci., 2013, 8, 8050-8057.

98 T. Alizadeh and S. Mirzagholipur, Sens. Actuators, B, 2014, 198, 438-447.

99 J. Song, L. Xu, C. Zhou, R. Xing, Q. Dai, D. Liu and H. Song, ACS Appl. Mater. Interfaces, 2013, 5, 12928-12934.

100 S. N. A. M. Yazid, I. M. Isa and N. Hashim, Mater. Sci. Eng., C, 2016, 68, 30575-30576.

101 J. Luo, H. Zhang, S. Jiang, J. Jiang and X. Liu, Microchim. Acta, 2012, 177, 485-490.

102 Y. Li, F. Huang, J. Chen, T. Mo, S. Li, F. Wang, S. Feng and Y. Li, Int. J. Electrochem. Sci., 2013, 8, 6332-6342.

103 L. Luo, L. Zhu and Z. Wang, Bioelectrochemistry, 2012, 156163.

104 C. L. Sun, W. L. Cheng, T. K. Hsu, C. W. Chang, J. L. Chang and J. M. Zen, Electrochem. Commun., 2013, 30, 91-94.

105 S. Muralikrishna, K. Sureshkumar, Z. Yan, C. Fernandez and T. Ramakrishnappa, J. Braz. Chem. Soc., 2015, 26, 1632-1641.

106 X. Yan, J. Yang, L. Ma, X. Tong, Y. Wang, G. Jin and X. Y. Guo, J. Solid State Electrochem., 2015, 19, 3195-3199.
107 L. T. Hoa, J. S. Chung and S. H. Hur, Sens. Actuators, B, 2016, 223, 76-82.

108 Y. Zheng, P. Li, H. Li and S. Chen, Int. J. Electrochem. Sci., 2014, 9, 7369-7381.

109 H. Heidari and E. Habibi, Microchim. Acta, 2016, 183, 22592266.

110 X. Wang, X. Dong, Y. Wen, C. Li, Q. Xiong and P. Chen, Chem. Commun., 2012, 48, 6490-6492.

111 S. J. Li, J. M. Du, J. Chen, N. N. Mao, M. J. Zhang and H. Pang, J. Solid State Electrochem., 2014, 18, 1049-1056.

112 M. Yang, J. M. Jeong, K. G. Lee, D. H. Kim, S. J. Lee and B. G. Choi, J. Biosci., 2016, 1, 75.

113 P. Lu, J. Yu, Y. Lei, S. Lu, C. Wang, D. Liu and Q. Guo, Sens. Actuators, B, 2015, 208, 90-98.

114 W. Lv, F. M. Jin, Q. Guo, Q. H. Yang and F. Kang, Electrochim. Acta, 2012, 73, 129-135.

115 J. Y. Sun, K. J. Huang, Y. Fan, Z. W. Wu and D. D. Li, Microchim. Acta, 2011, 174, 289-294.

116 H. Belkhalfa, F. Teodorescu, G. Quéniat, Y. Coffinier, N. Dokhan, S. Sam, A. Abderrahmani, R. Boukherroub and S. Szunerits, Sens. Actuators, B, 2016, 237, 693-701.

117 Y. Zhang, Y. Wang, J. Jia and J. Wang, Sens. Actuators, B, 2012, 171-172, 580-587.

118 P. Subramanian, J. N. Jonsson, A. Lesniewski, Q. Wang, M. Li, R. Boukherroub and S. Szunerits, J. Mater. Chem. A, 2014, 2, 5525-5533.

119 V. A. Kumary, T. E. M. Nancy, J. Divya and K. Sreevalsan, Int. J. Electrochem. Sci., 2013, 8, 2220-2228.

120 H. Zhang and S. Liu, Sens. Actuators, B, 2017, 238, 788-794.

121 Y. Zhang, F. Xu, Y. Sun, Y. Shi, Z. Wen and Z. Li, J. Mater. Chem., 2011, 21, 16949.

122 N. Qiao and J. Zheng, Microchim. Acta, 2012, 177, 103-109.

123 X. Zhu, Q. Jiao, C. Zhang, X. Zuo, X. Xiao, Y. Liang and J. Nan, Microchim. Acta, 2013, 180, 477-483.

124 Y. Ye, P. Wang, E. Dai, J. Liu, Z. Tian, C. Liang and G. Shao, Phys. Chem. Chem. Phys., 2014, 16, 8801.

125 H. Gao, F. Xiao, C. B. Ching and H. Duan, ACS Appl. Mater. Interfaces, 2011, 3, 3049-3057.

126 F. Xiao, Y. Li, H. Gao, S. Ge and H. Duan, Biosens. Bioelectron., 2013, 41, 417-423.

127 L. Wang, X. Lu, Y. Ye, L. Sun and Y. Song, Electrochim. Acta, 2013, 114, 484-493.

128 K. Dhara, J. Stanley, T. Ramachandran, B. G. Nair and S. Babu, Sens. Actuators, B, 2014, 195, 197-205.

129 M. Li, X. Bo, Z. Mu, Y. Zhang and L. Guo, Sens. Actuators, B, 2014, 192, 261-268.

130 M. Yuan, A. Liu, M. Zhao, W. Dong, T. Zhao, J. Wang and W. Tang, Sens. Actuators, B, 2014, 190, 707-714.

131 Y. Hu, F. He, A. Ben and C. Chen, J. Electroanal. Chem., 2014, 726, 55-61.

132 M. Li, X. Bo, Y. Zhang, C. Han and L. Guo, Biosens. Bioelectron., 2014, 56, 223-230.

133 X. Chen, X. Tian, L. Zhao, Z. Huang and M. Oyama, Microchim. Acta, 2014, 181, 783-789.

134 A. A. Ensafi, Z. Ahmadi, M. J. Asl and B. Rezaei, Electrochim. Acta, 2015, 173, 619-629. 
135 K. Dhara, T. Ramachandran, B. G. Nair and T. G. Babu, J. Electroanal. Chem., 2015, 743, 1-9.

136 K. Dhara, T. Ramachandran, B. G. Nair and G. S. Babu, Microchim. Acta, 2015, 182, 2183-2192.

137 G. Li, H. Huo and C. Xu, J. Mater. Chem. A, 2015, 3, 4922.

138 L. M. Lu, H. B. Li, F. Qu, X. B. Zhang, G. L. Shen and R. Q. Yu, Biosens. Bioelectron., 2011, 26, 3500-3504.

139 J. Yang, J. H. Yu, J. Rudi Strickler, W. J. Chang and S. Gunasekaran, Biosens. Bioelectron., 2013, 47, 530-538.

140 Z. Wang, J. Xia, X. Qiang, Y. Xia, G. Shi, F. Zhang, G. Han, L. Xia and J. Tang, Int. J. Electrochem. Sci., 2013, 8, 69416950.

141 L. Wang, Y. Zheng, X. Lu, Z. Li, L. Sun and Y. Song, Sens. Actuators, B, 2014, 195, 1-7.
142 N. Hui, S. Wang, H. Xie, S. Xu, S. Niu and X. Luo, Sens. Actuators, B, 2015, 221, 606-613.

143 M. M. Farid, L. Goudini, F. Piri, A. Zamani and F. Saadati, Food Chem., 2016, 194, 61-67.

144 D. Jiang, Q. Liu, K. Wang, J. Qian, X. Dong, Z. Yang, X. Du and B. Qiu, Biosens. Bioelectron., 2014, 54, 273-278.

145 J. Balamurugan, T. D. Thanh, S. B. Heo, N. H. Kim and J. H. Lee, Carbon, 2015, 94, 962-970.

146 S. Yang, G. Li, G. Wang, J. Zhao, X. Gao and L. Qu, Sens. Actuators, B, 2015, 221, 172-178.

147 S. Yang, L. Liu, G. Wang, G. Li, D. Deng and L. Qu, J. Electroanal. Chem., 2015, 755, 15-21.

148 Y. Tian, Y. Liu, W. Wang, X. Zhang and W. Peng, Electrochim. Acta, 2015, 156, 244-251. 